\title{
ASYMPTOTICS FOR THE MOMENTS OF THE OVERSHOOT AND UNDERSHOOT OF A RANDOM WALK
}

\author{
ZHAOLEI CUI* AND \\ YUEBAO WANG, ${ }^{* * *}$ Soochow University \\ KAIYONG WANG, ${ }^{* * *}$ Suzhou University of Science and Technology
}

\begin{abstract}
In this paper we obtain some equivalent conditions and sufficient conditions for the local and nonlocal asymptotics of the $\varphi$-moments of the overshoot and undershoot of a random walk, where $\varphi$ is a nonnegative, long-tailed function. By the strong Markov property, it can be shown that the moments of the overshoot and undershoot and the moments of the first ascending ladder height of a random walk satisfy some renewal equations. Therefore, in this paper we first investigate the local and nonlocal asymptotics for the moments of the first ascending ladder height of a random walk, and then give some equivalent conditions and sufficient conditions for the asymptotics of the solutions to some renewal equations. Using the above results, the main results of this paper are obtained.
\end{abstract}

Keywords: Local and nonlocal asymptotics; moment; first ascending ladder height; renewal equation; overshoot; undershoot

2000 Mathematics Subject Classification: Primary 60K05

Secondary 60K10; 60K30

\section{Introduction}

Assume that $\left\{X_{i}, i \geq 1\right\}$ is a sequence of independent, identically distributed (i.i.d.) random variables (RVs) with common distribution $F$ and finite mean $\mu_{F}=-m<0$. Set $S_{0}=0$ and $S_{n}=\sum_{i=1}^{n} X_{i}, n \geq 1$. Then $\left\{S_{n}, n \geq 0\right\}$ is a random walk generated by $\left\{X_{i}, i \geq 1\right\}$. For a level $x \geq 0$, denote the first passage time over $x$ by

$$
\tau_{x}=\inf \left\{n \geq 1: S_{n}>x\right\},
$$

where, by convention, inf $\varnothing=\infty$. Then $S_{\tau_{x}}-x$ is the overshoot and $x-S_{\tau_{x}-1}$ is the undershoot of the random walk at level $x$.

It is well known that the overshoot and undershoot are among the main features of random walks and they have many applications in risk theory, queueing theory, branching process theory, etc. There is considerable literature on the overshoot and undershoot of random walks. Janson (1986) gave necessary and sufficient conditions for the existence of moments for the first passage and last exit times, and related quantities for random walks with positive drift. Borovkov and Foss (2000) presented estimates for the magnitude of the overshoot over an arbitrary boundary and the weak convergence of the distribution of the overshoot. Klüppelberg et al. (2004)

Received 28 February 2008; revision received 13 April 2009.

* Postal address: Department of Mathematics, Soochow University, Suzhou, 215006, P. R. China.

** Email address: ybwang@suda.edu.cn

*** Postal address: Department of Information and Computational Science, School of Mathematics and Physics, Suzhou University of Science and Technology, Suzhou, 215009, P. R. China. 
formulated the insurance risk process in a general Lévy process setting, and gave general theorems for the ruin probability and the asymptotic distribution of the overshoot of the process above a high level. Doney and Kyprianou (2006) used a new fluctuation identity for a general Lévy process and gave the asymptotic overshoot distribution of a Lévy process. Tang (2007) obtained uniform asymptotics of the overshoot of a random walk with negative drift, and the local uniform asymptotics of the overshoot of a random walk have been investigated in Chen $e t$ al. (2008). Many of the aforementioned works focus on the study of the asymptotics for the distributions of the overshoot and undershoot of a random walk. In this paper we will investigate the local and nonlocal asymptotics for the moments of the overshoot and undershoot of a random walk. These results indicate the average asymptotics of the overshoot and undershoot of a random walk. In particular, the asymptotics of the corresponding distribution can be given.

Throughout this paper, we assume that $\varphi$ is a nonnegative function supported on $[0, \infty)$. For any $0<T \leq \infty$, set $\triangle_{T}=(0, T]$ and $x+\triangle_{T}=(x, x+T]$; if $T=\infty$ then let $\triangle_{\infty}=(0, \infty)$ and $x+\triangle_{\infty}=(x, \infty)$. When $0<T<\infty$, the asymptotics of

$$
\mathrm{E} \varphi\left(S_{\tau_{x}}-x\right) \mathbf{1}_{\left\{S_{\tau_{x}} \in x+\triangle_{T}\right\}} \quad \text { and } \quad \mathrm{E} \varphi\left(x-S_{\tau_{x}-1}\right) \mathbf{1}_{\left\{S_{\tau_{x}} \in x+\triangle_{T}\right\}}
$$

are called the local asymptotics for the $\varphi$-moments of the overshoot and undershoot of a random walk; when $T=\infty$, they are called the nonlocal asymptotics for the $\varphi$-moments of the overshoot and undershoot of a random walk.

We first recall a related result. Using the theory of ascending ladder heights, Cheng et al. (2002) obtained nonlocal asymptotics for the $\varphi$-moments of the deficit at the ruin time in the renewal risk model. In other words, they obtained nonlocal asymptotics for $\mathrm{E} \varphi\left(S_{\tau_{x}}-x\right) \mathbf{1}_{\left\{\tau_{x}<\infty\right\}}$, where they required the condition that $\varphi$ is nondecreasing, which made it convenient for them to prove their results using integration by parts. Also, using the nondecreasing property of $\varphi$, a simple proof of the results of Cheng et al. (2002) for the heavy-tailed case has been given (see Cui and Wang (2007)). But, in order to extend the scope of applications and also for the mathematical interest, in this paper we will let $\varphi$ be a long-tailed function (see the definition below), which may not be nondecreasing. In this paper we will also consider the undershoot of a random walk, and we will investigate not only the nonlocal asymptotics but also the local asymptotics for the $\varphi$-moments of the overshoot and undershoot of a random walk with heavytailed increments. Since the overshoot, $S_{\tau_{x}}-x$, and the undershoot, $x-S_{\tau_{x}-1}$, may often not be very large in practice, the latter is more interesting than the former.

Since $\varphi$ may not be nondecreasing, we cannot use the method of Cheng et al. (2002). We will use the renewal equations to prove our results. Using the strong Markov property, we find that the moments of the overshoot and undershoot and the moments of the first ascending ladder height of a random walk satisfy some renewal equations. So we first consider the local and nonlocal asymptotics for the moments of the first ascending ladder height of a random walk, and then give some equivalent conditions and sufficient conditions for the asymptotics of the solutions to some renewal equations. On the basis of the above results, some equivalent conditions and sufficient conditions for the local and nonlocal asymptotics of the moments of the overshoot and undershoot of a random walk are obtained. For example, under some mild conditions, Theorem 2.1, below, presents some equivalent conditions for the following local asymptotics:

$$
\lim _{x \rightarrow \infty} \frac{\mathrm{E} \varphi\left(S_{\tau_{x}}-x\right) \mathbf{1}_{\left\{S_{\tau_{x}} \in x+\triangle_{T}\right\}}}{\bar{F}(x)}=C_{0},
$$

where $C_{0}$ is a positive constant. In particular, when $\varphi \equiv 1$, the above result can describe the local asymptotics of the distribution of the overshoot. Thus, it can describe the asymptotics of 
the local ruin probability in the renewal risk model (see Remark 2.2, below). The results of this paper can also be applied to other fields of applied probability.

Recently, Park and Maller (2008) considered the nonlocal asymptotics of the $\beta$-order moment of the overshoot and undershoot of a Lévy process with drifts to $-\infty$ almost surely (here $\beta$ is a positive constant). Since a Lévy process is different from a random walk, in forthcoming papers we will investigate the uniform local asymptotics of a Lévy process and its overshoot and undershoot, and the local asymptotics of the $\varphi$-moments of the overshoot and undershoot of a Lévy process.

The paper is organized as follows. In Section 2 we will present our main results, i.e. equivalent conditions and sufficient conditions for the local and nonlocal asymptotics of the moments of the overshoot and undershoot of a random walk. In Section 4 we will investigate the local and nonlocal asymptotics of the moments of the first ascending ladder height of a random walk. For this, in Section 3, two general results about asymptotics of the moments of a random walk will be given. In Section 5 we will give some equivalent conditions and sufficient conditions for the asymptotics of the solutions to some renewal equations. Using the results of Sections 4 and 5, the main results of this paper will be proved in Section 6 .

\section{Main results}

In order to give the main results of this paper, we will introduce some notions and notation, which will be valid throughout the rest of this paper.

Unless stated otherwise, in this paper a limit is taken as $x \rightarrow \infty$ and, for a given function or distribution, we assume that its support is $D=(-\infty, \infty)$ or $[0, \infty)$. Let $f_{1}(x)$ and $f_{2}(x)$ be nonnegative functions. We write $f_{1}(x) \sim f_{2}(x)$ if $\lim f_{1}(x) / f_{2}(x)=1$; $f_{1}(x)=O(1) f_{2}(x)$ if limsup $f_{1}(x) / f_{2}(x)<\infty ; f_{1}(x) \lesssim f_{2}(x)$ if $\lim \sup f_{1}(x) / f_{2}(x) \leq 1$; $f_{1}(x) \approx f_{2}(x)$ if $f_{1}(x)=O(1) f_{2}(x)$ and $f_{2}(x)=O(1) f_{1}(x)$; and $f_{1}(x)=o(1) f_{2}(x)$ if $\lim f_{1}(x) / f_{2}(x)=0$. For a distribution $V$, let $\bar{V}=V(\infty)-V$ and let $V^{I}(x)=V(\infty)-$ $\min \left\{V(\infty), \int_{x}^{\infty} \bar{V}(y) \mathrm{d} y\right\}, x \geq 0$, be the integrated distribution of $V$. Denote the $n$-fold convolution of $V$ by $V^{* n}, n=0,1,2, \ldots$, where $V^{* 1}=V$ and $V^{* 0}$ is the distribution degenerated at 0 .

Since asymptotics of the moments of the overshoot and undershoot of a random walk have a close relation with the corresponding distribution, we will introduce some function classes and distribution classes. We say that a nonnegative function $f \in \mathcal{L} d(\gamma)$ for some $\gamma \geq 0$ if $f$ is eventually positive and, for all $y \in(-\infty, \infty)$,

$$
f(x-y) \sim \mathrm{e}^{\gamma y} f(x) .
$$

We say that a nonnegative function $f \in \delta d(\gamma)$ for some $\gamma \geq 0$ if

$$
f \in \mathcal{L} d(\gamma), \quad 0<a=\int_{0}^{\infty} f(y) \mathrm{d} y<\infty, \quad \text { and } \quad \int_{0}^{x} f(x-y) f(y) \mathrm{d} y \sim 2 a f(x) .
$$

In particular, we call $\mathcal{L} d(0)$ the long-tailed function class and $\delta d(0)$ the subexponential function class, denoted by $\mathcal{L} d$ and $\delta d$, respectively. For the properties and applications of $\mathcal{L} d(\gamma)$ and $s d(\gamma)$, we refer the reader to Klüppelberg (1989), Wang and Wang (2006), and Wang and Wang (2009), among others.

We say that a distribution $V \in \mathcal{L}(\gamma)$ for some $\gamma \geq 0$ if $\bar{V} \in \mathcal{L} d(\gamma)$. We say that a proper distribution $V \in \delta(\gamma)$ for some $\gamma \geq 0$ if $V \in \mathcal{L}(\gamma)$ and there exists a positive constant $c$ such that $\overline{V^{* 2}}(x) \sim 2 c \bar{V}(x)$. When $V$ is defective, i.e. $V(\infty)<1$, we say that $V \in \delta(\gamma)$ 
for some $\gamma \geq 0$ if $V / V(\infty) \in s(\gamma)$. Let $g_{V}(-s)=\int_{D} \mathrm{e}^{s y} V(\mathrm{~d} y)$ for any $s \geq 0$. Foss and Korshunov (2007) have shown that $c=g_{V}(-\gamma)$ for the case in which $D=[0, \infty)$. Recently, Yu et al. (2009) obtained some corresponding results for the case in which $D=(-\infty, \infty)$. We call $\delta(\gamma), \gamma \geq 0$, the convolution equivalent distribution class. Specifically, we call $\mathcal{L}(0)$ the long-tailed distribution class and $\delta(0)$ the subexponential distribution class, denoted by $\mathcal{L}$ and $\S$, respectively. The class $\delta(\gamma)$ was introduced in Chistyakov (1964) and Chover et al. (1973a), (1973b) for distributions on [0, $\infty)$, and in Pakes (2004) for distributions on $(-\infty, \infty)$. Bertoin and Doney (1996) pointed out that in the definition of $\mathcal{L}(\gamma)$, when $\gamma>0$ and $V$ is a lattice distribution, $x$ and $y$ should be taken to be an integer multiple of the span, which we will assume to be the case in the following. In addition, Klüppelberg (1988) introduced a subclass of $\delta$, denoted by $\delta^{*}$. We say that a distribution $V \in \delta^{*}$ if $\bar{V} \in \delta d$. Klüppelberg (1988) showed that if a distribution $V \in \delta^{*}$ then $V \in \delta$ and $V^{I} \in \delta$. But Denisov et al. (2004) pointed out that the converse proposition is not true, that is to say there exists a distribution $V$ with finite mean such that $V \in \delta$ and $V^{I} \in \delta$, but $V \notin \varsigma^{*}$.

Asmussen et al. (2003) introduced some local distribution classes. For a distribution $V$, let $g(x)=V\left(x+\triangle_{T}\right)$ for some $0<T \leq \infty$. We say that $V \in \mathcal{L}_{\triangle_{T}}$ if $g \in \mathcal{L} d$. For a proper distribution $V$ on $[0, \infty)$, we say that $V \in \delta_{\triangle_{T}}$ if $V \in \mathcal{L}_{\triangle_{T}}$ and $V^{* 2}\left(x+\triangle_{T}\right) \sim 2 V\left(x+\triangle_{T}\right)$. When $V$ is defective, i.e. $V(\infty)<1$, we say that $V \in s_{\triangle_{T}}$ if $V / V(\infty) \in s_{\triangle_{T}}$. Asmussen et al. (2003) called $\mathcal{L}_{\Delta_{T}}$ the local long-tailed distribution class and $\delta_{\triangle_{T}}$ the local subexponential distribution class. Asmussen et al. (2003) systematically investigated the properties of $\mathscr{L}_{\triangle_{T}}$ and $\varsigma_{\triangle_{T}}$, and gave applications to random walks, the key renewal theorem, the compound Poisson process, infinitely divisible laws, and Bellman-Harris branching processes. Furthermore, Wang et al. (2005) investigated the closure of the local subexponential distribution class under convolution roots and gave an application to infinitely divisible laws. Denisov and Shneer (2007) obtained local asymptotics of the cycle maximum of a random walk with local subexponential increments. Using the local subexponential distribution class, Wang et al. (2007) studied the local asymptotics for random sums and gave applications to infinitely divisible laws. Chen $e t$ al. (2008) obtained uniform local asymptotics of the distribution of the overshoot of a random walk with heavy-tailed increments. Gao and Wang (2009) obtained equivalent conditions for the asymptotics of the local ruin probability in the random multi-delayed renewal risk model, among others. In the above studies, these local distribution classes are often not only the sufficient condition but also the necessary condition for the local asymptotics.

Recall that $\tau_{x}, x \geq 0$, is given in Section 1. In particular, write $\tau_{+}=\tau_{0}$. We call $\tau_{+}$the first ascending ladder epoch and $S_{\tau_{+}}$the first ascending ladder height of the random walk. As $\tau_{+}$, we denote the first weak descending ladder epoch of the random walk by

$$
\tau_{-}=\inf \left\{n \geq 1: S_{n} \leq 0\right\}
$$

and call $S_{\tau_{-}}$the first weak descending ladder height. Let $F_{+}$and $F_{-}$be the distributions of $S_{\tau_{+}}$and $S_{\tau_{-}}$, respectively. It is well known (see, e.g. Asmussen (2003, Chapter VIII)) that, when $m>0, F_{-}$is proper and $F_{+}$is defective, i.e. $\alpha=F_{+}(\infty)=1-\mathrm{e}^{-B}<1$, where $B=\sum_{n=1}^{\infty} n^{-1} \mathrm{P}\left(S_{n}>0\right)<\infty$. In this case we define the proper distribution of $F_{+}$by

$$
F_{p+}(x)=\mathrm{P}\left(S_{\tau_{+}} \leq x \mid \tau_{+}<\infty\right)=\alpha^{-1} F_{+}(x), \quad x \geq 0 .
$$

For any $s \geq 0$, set $g_{+}(-s)=g_{F_{+}}(-s)$ and $g_{-}(-s)=g_{F_{-}}(-s)$. 
In the following we give some equivalent conditions and sufficient conditions for the local and nonlocal asymptotics of the $\varphi$-moments of the overshoot and undershoot of a random walk. They will be presented for the heavy-tailed and light-tailed cases.

\subsection{Heavy-tailed case}

We first give the local asymptotic results, which are the main results of this section.

Theorem 2.1. Assume that $\varphi \in \mathcal{L} d$, that $\varphi$ is continuous and satisfies the conditions

$$
\int_{0}^{\infty} \varphi(y) \bar{F}(y) \mathrm{d} y<\infty \text { and } \varphi(x)=O(1) \inf _{y \geq x} \varphi(y),
$$

and that, for any $a>0$,

$$
\bar{\varphi}_{a} \equiv \sup _{y \in[0, a]} \varphi(y)<\infty .
$$

Also, let $F \in \mathcal{L}$. Then the following assertions are equivalent:

(i) $F \in 8^{*}$;

(ii) $F^{I} \in \varsigma_{\triangle_{T}}$ for all $0<T<\infty$;

(iii) $U_{0} \in \mathcal{L}, F \in \mathcal{L}, \overline{F^{I}}(x) \approx \overline{U_{0}}(x)$, and

$$
\begin{aligned}
\operatorname{E} \varphi\left(S_{\tau_{x}}-x\right) \mathbf{1}_{\left\{S_{\tau_{x}} \in x+\triangle_{T}\right\}} & \sim\left(I T^{-1}(1-\alpha)^{-1}+C\right) m^{-1} F^{I}\left(x+\triangle_{T}\right) \\
& \sim\left(I(1-\alpha)^{-1}+C T\right) m^{-1} \bar{F}(x) \text { for all } 0<T<\infty,
\end{aligned}
$$

where

$$
I=\int_{0}^{\infty} \mathrm{E} \varphi\left(S_{\tau_{+}}-y\right) \mathbf{1}_{\left\{S_{\tau_{+}} \in y+\triangle_{T}\right\}} \mathrm{d} y \text { and } C=T^{-1} \int_{0}^{T} \varphi(y) \mathrm{d} y .
$$

Here we note that for (ii) to imply (i) we only need $F^{I} \in s_{\triangle_{T}}$ for some $0<T<\infty$.

Theorem 2.2. Assume that $\varphi \in \mathcal{L} d$, that $\varphi(x) \rightarrow a$, and that $\varphi$ satisfies (2.1). Also, let $F \in \mathcal{L}_{\triangle_{T_{1}}}$ for some $0<T_{1}<\infty$, and define $z_{1}(x)=\mathrm{E} \varphi\left(x-S_{\tau_{+}-1}\right) \mathbf{1}_{\left\{S_{\tau_{+}} \in x+\triangle_{T_{1}}\right\}}, x \geq 0$.

Case 1. If $0<a<\infty, F \in \mathcal{S}^{*}$, and $z_{1}(x)$ is directly Riemann integrable, then

$$
\begin{aligned}
\mathrm{E} \varphi\left(x-S_{\tau_{x}-1}\right) \mathbf{1}_{\left\{S_{\tau_{x}} \in x+\triangle_{T_{1}}\right\}} & \sim\left((1-\alpha)^{-1} I T_{1}^{-1}+a\right) m^{-1} F^{I}\left(x+\triangle_{T_{1}}\right) \\
& \sim\left((1-\alpha)^{-1} I+a T_{1}\right) m^{-1} \bar{F}(x),
\end{aligned}
$$

where $I=\int_{0}^{\infty} z_{1}(y) \mathrm{d} y$.

Case 2. If $a=\infty$ and $z_{1} \in \& d$, then

$$
\mathrm{E} \varphi\left(x-S_{\tau_{x}-1}\right) \mathbf{1}_{\left\{S_{\tau_{x}} \in x+\triangle_{T_{1}}\right\}} \sim m^{-1} \int_{x}^{\infty} \varphi(y) F\left(y+\triangle_{T_{1}}\right) \mathrm{d} y .
$$

Remark 2.1. If $\varphi \equiv 1$ in Theorem 2.1 or case 1 of Theorem 2.2, then an interesting result can be obtained, i.e.

$$
\mathrm{P}\left(S_{\tau_{x}} \in x+\triangle_{T_{1}}\right) \sim\left((1-\alpha)^{-1} I+T_{1}\right) m^{-1} \bar{F}(x),
$$

where $I=\alpha \int_{0}^{T_{1}} \bar{F}_{p+}(y) \mathrm{d} y=\int_{0}^{T_{1}} \bar{F}_{+}(y) \mathrm{d} y$. From (2.5) we find that the above local asymptotics are different from the local asymptotics for the supremum of the random walk. 
Denote the supremum of a random walk by $M$. Asmussen et al. (2002) have shown that if $F \in \delta^{*}$ then

$$
\mathrm{P}\left(M \in x+\triangle_{T_{1}}\right) \sim T_{1} m^{-1} \bar{F}(x) .
$$

Foss and Zachary (2003, Theorem 1) pointed out that $F \in 8^{*}$ is also necessary for (2.6). By (2.5) and (2.6), we know that $\mathrm{P}\left(S_{\tau_{x}} \in x+\triangle_{T_{1}}\right)$ is larger than $\mathrm{P}\left(M \in x+\triangle_{T_{1}}\right)$ eventually. But Wang et al. (2008) showed that (2.5) is equivalent to $F \in 8^{*}$. Thus, (2.5) is equivalent to (2.6).

Remark 2.2. In the renewal risk model, assume that in an insurance company the claim sizes are i.i.d. $\mathrm{RVs}\left\{\xi_{i}, i \geq 1\right\}$ with finite mean $\mathrm{E} \xi_{1}$, and that the claim interarrival times are also i.i.d. RVs $\left\{\eta_{i}, i \geq 1\right\}$ with finite mean $\mathrm{E} \eta_{1}$, which are independent of $\left\{\xi_{i}, i \geq 1\right\}$. Let $x$ be the initial capital of the insurance company. Let the constant $c$ be the so-called premium income rate that satisfies the safety loading condition $c \mathrm{E} \eta_{1}-\mathrm{E} \xi_{1}>0$. Let $X_{i}=\xi_{i}-c \eta_{i}, i \geq 1$, and let $M=\sup _{n \geq 0} S_{n}$. The number of claims in the interval $[0, t]$ is denoted by

$$
N(t)=\sup \left\{n \geq 1: \sum_{i=1}^{n} \eta_{i} \leq t\right\}, \quad t \geq 0,
$$

where, by convention, sup $\varnothing=0$. It is well known (see, e.g. Embrechts et al. (1997, Chapter 1)) that the infinite-time ruin probability is

$$
\begin{aligned}
\psi(x) & =\mathrm{P}\left(\sup _{t \geq 0}\left(\sum_{i=1}^{N(t)} \xi_{i}-c t\right)>x\right) \\
& =\mathrm{P}\left(\tau_{x}<\infty\right) \\
& =\mathrm{P}(M>x) .
\end{aligned}
$$

For some $0<T<\infty$, let

$$
\psi^{T}(x)=\mathrm{P}\left(S_{\tau_{x}} \in x+\triangle_{T}\right) .
$$

Hence, by (2.5) and (2.6), we find that $\psi^{T}(x)$ is larger than $\mathrm{P}\left(M \in x+\triangle_{T}\right)$ eventually, and we think that it is reasonable to use $\psi^{T}(x)$ to define the local ruin probability of an insurance company. It follows from Theorem 2(B) of Veraverbeke (1977) or (2.8), below, that

$$
\psi(x) \sim m^{-1} \overline{F^{I}}(x) .
$$

Hence, $\psi^{T}(x)=o(1) \psi(x)$. Also, as above, the deficit at the ruin time, i.e. the overshoot $S_{\tau_{x}}-x$, is often not very large in practice. Therefore, it is necessary to investigate the local ruin probability $\psi^{T}(x)$. The results of this paper, especially (2.5), give the asymptotics of the local ruin probability.

The following results are the nonlocal asymptotic results.

Theorem 2.3. Assume that $\varphi \in \mathcal{L} d$, that $\varphi(x) \rightarrow a$, and that $\varphi$ satisfies (2.1) and (2.2). Also, let $F^{I} \in \mathcal{L}_{\triangle_{T}}$ for some $0<T<\infty$.

Case 1. If $0<a<\infty$ then the following assertions are equivalent:

$$
\text { (i) } F^{I} \in s ; \quad \text { (ii) } \mathrm{E} \varphi\left(S_{\tau_{x}}-x\right) \mathbf{1}_{\left\{\tau_{x}<\infty\right\}} \sim a m^{-1} \overline{F^{I}}(x) .
$$

Case 2. If $a=\infty$ and $G \in$ s, where $\bar{G}(x)=\min \left\{1, \int_{0}^{\infty} \varphi(y) \bar{F}(x+y) \mathrm{d} y\right\}, x \geq 0$, then

$$
\mathrm{E} \varphi\left(S_{\tau_{x}}-x\right) \mathbf{1}_{\left\{\tau_{x}<\infty\right\}} \sim m^{-1} \int_{0}^{\infty} \varphi(y) \bar{F}(x+y) \mathrm{d} y .
$$


Theorem 2.4. Assume that $\varphi \in \mathcal{L} d$, that $\varphi(x) \rightarrow a$, and that $\varphi$ satisfies (2.1). Also, let $F^{I} \in \mathcal{L}$.

Case 1. If $0<a<\infty$ then the following assertions are equivalent:

$$
\text { (i) } F^{I} \in \& ; \quad \text { (ii) } \operatorname{E} \varphi\left(x-S_{\tau_{x}-1}\right) \mathbf{1}_{\left\{\tau_{x}<\infty\right\}} \sim a m^{-1} \overline{F^{I}}(x) .
$$

Case 2. If $a=\infty$ and $G \in 8$, where $\bar{G}(x)=\min \left\{1, \int_{x}^{\infty} \varphi(y) \bar{F}(y) \mathrm{d} y\right\}, x \geq 0$, then

$$
\operatorname{E} \varphi\left(x-S_{\tau_{x}-1}\right) \mathbf{1}_{\left\{\tau_{x}<\infty\right\}} \sim m^{-1} \int_{x}^{\infty} \varphi(y) \bar{F}(y) \mathrm{d} y .
$$

Remark 2.3. We find that $\mathrm{E} \varphi\left(S_{\tau_{x}}-x\right) \mathbf{1}_{\left\{\tau_{x}<\infty\right\}}$ and $\mathrm{E} \varphi\left(x-S_{\tau_{x}-1}\right) \mathbf{1}_{\left\{\tau_{x}<\infty\right\}}$ in case 1 of Theorems 2.3 and 2.4 have the same asymptotics. But the conditions of Theorem 2.4 are weaker than those of Theorem 2.3.

\subsection{Light-tailed case}

It is well known that in the light-tailed case, it is not necessary to investigate the local asymptotics. Therefore, in this subsection we will give only the nonlocal asymptotics for the moments of the overshoot and undershoot in the light-tailed case.

Theorem 2.5. Assume that $\varphi \in \mathcal{L} d$, that $\int_{0}^{\infty} \varphi(y) \bar{F}(y) \mathrm{d} y<\infty$, and that $\varphi$ is continuous. Also, assume that $F$ has a density $f \in \mathcal{L} d(\gamma)$ for some $0<\gamma<\infty$, that $g_{F}(-\gamma)<1$, and that $\bar{F}(x) \mathrm{e}^{\gamma x} \bar{\downarrow}$, i.e. there exists a positive constant $C$ (independent of $x$ ) such that, for any $y \geq x$,

$$
\bar{F}(y) \mathrm{e}^{\gamma y} \leq C \bar{F}(x) \mathrm{e}^{\gamma x} .
$$

Then the following assertions are equivalent:

(i) $F \in \delta(\gamma)$;

(ii) $F \in \mathcal{L}(\gamma)$ and

$$
\begin{aligned}
\operatorname{E} \varphi\left(S_{\tau_{x}}-x\right) \mathbf{1}_{\left\{\tau_{x}<\infty\right\}} & \\
& \sim\left(\gamma \int_{0}^{\infty} \varphi(y) \mathrm{e}^{-\gamma y} \mathrm{~d} y+I\left(1-g_{+}(-\gamma)\right)^{-1}\right)\left(1-g_{F}(-\gamma)\right)^{-1} \bar{F}(x),
\end{aligned}
$$

where $I=\gamma \int_{0}^{\infty} \mathrm{e}^{\gamma y} \mathrm{E} \varphi\left(S_{\tau_{+}}-y\right) \mathbf{1}_{\left\{S_{\tau_{+}}>y\right\}} \mathrm{d} y$.

Theorem 2.6. Assume that $\varphi \in \mathcal{L} d$, that $\varphi(x) \rightarrow a$, and that $\int_{0}^{\infty} \varphi(y) \bar{F}(y) \mathrm{d} y<\infty$. Also, let $F \in \mathcal{L}(\gamma)$ for some $0<\gamma<\infty$ and let $g_{F}(-\gamma)<1$.

Case 1. If $0<a<\infty$ then the following assertions are equivalent:

(i) $F \in \delta(\gamma)$;

(ii) $F \in \mathcal{L}(\gamma)$ and

$$
\mathrm{E} \varphi\left(x-S_{\tau_{x}-1}\right) \mathbf{1}_{\left\{\tau_{x}<\infty\right\}} \sim\left(a+I\left(1-g_{+}(-\gamma)\right)^{-1}\right)\left(1-g_{F}(-\gamma)\right)^{-1} \bar{F}(x),
$$

where $I=\gamma \int_{0}^{\infty} \mathrm{e}^{\gamma y} \mathrm{E} \varphi\left(y-S_{\tau_{+}-1}\right) \mathbf{1}_{\left\{S_{\tau_{+}}>y\right\}} \mathrm{d} y$.

Case 2. If $a=\infty$ and $G \in \varsigma(\gamma)$, where $\bar{G}(x)=\min \left\{1, \int_{x}^{\infty} \varphi(y) \bar{F}(y) \mathrm{d} y\right\}, x \geq 0$, then

$$
\mathrm{E} \varphi\left(x-S_{\tau_{x}-1}\right) \mathbf{1}_{\left\{\tau_{x}<\infty\right\}} \sim\left(1-g_{F}(-\gamma)\right)^{-1} \varphi(x) \bar{F}(x) .
$$




\section{Two lemmas}

In order to investigate asymptotics for the moments of the first ascending ladder height of a random walk, we will give two general results about asymptotics for the moments of a random walk as lemmas in this section. The first lemma is Proposition 3.1 of Wang and Wang (2006), which is inspired by Lemma 3 of Asmussen et al. (2003). For any given function $u$ and some $0<T \leq \infty$, let $u_{T}(x)=u(x)-u(x+T), U(x)=\int_{x}^{\infty} u(y) \mathrm{d} y$, and $U_{T}(x)=$ $U(x)-U(x+T), x \in(-\infty, \infty)$. Recall that the random walk $\left\{S_{n}, n \geq 0\right\}$ is given in Section 1. Let $\Lambda_{n}=\left\{S_{j} \leq 0: 0 \leq j \leq n\right\}, n \geq 1$ and $\Lambda_{0}=\Omega$.

Lemma 3.1. (Wang and Wang (2006, Proposition 3.1).) Assume that $U(0)<\infty$ and $U_{T} \in$ $\mathcal{L} d$; that $u$ on $(-\infty, \infty)$ is an eventually positive, nonincreasing function for some $0<T<\infty$; and that $u(x)=u_{1}(x) u_{2}(x), x \in(-\infty, \infty)$, for $T=\infty$, where $u_{1} \in \mathcal{L} d$ and $u_{2}$ is an eventually positive, nonincreasing function. Then

$$
I(x)=\sum_{n=0}^{\infty} \mathrm{E} u_{T}\left(x-S_{n}\right) \mathbf{1}_{\Lambda_{n}} \sim(1-\alpha) m^{-1} U_{T}(x) .
$$

Now we give a light-tailed version of Lemma 3.1.

Lemma 3.2. For some $\gamma>0$, let $g_{F}(-\gamma)<1$. Assume that the function $l$ on $D$ belongs to $\mathcal{L} d(\gamma)$ and that $\int_{0}^{\infty} l(y) \mathrm{d} y<\infty$. Then

$$
I(x)=\sum_{n=0}^{\infty} \mathrm{E} l\left(x-S_{n}\right) \mathbf{1}_{\Lambda_{n}} \sim\left(1-g_{-}(-\gamma)\right)^{-1} l(x),
$$

and, thus, $I \in \mathcal{L} d(\gamma)$.

Proof. It follows from $l \in \mathcal{L} d(\gamma)$ for some $\gamma>0$ and Karamata's theorem that

$$
l(x) \sim \gamma \int_{x}^{\infty} l(y) \mathrm{d} y .
$$

We consider the taboo renewal function $H(B)=\sum_{n=0}^{\infty} H_{n}(B)$ for the set $B \subset(-\infty, 0]$, where $H_{0}(B)=\mathbf{1}_{\{0 \in B\}}$ and $H_{n}(B)=\mathrm{P}\left(\Lambda_{n}, S_{n} \in B\right)$ for all $n \geq 1$. It follows from Theorem 2.3(b) of Asmussen (2003) that

$$
H(B)=\sum_{n=0}^{\infty}\left(F_{-}\right)^{* n}(B) .
$$

Since $g_{F}(-\gamma)<1$, by Wiener-Hopf factorization, we obtain $g_{-}(-\gamma)<1$. Hence, by (3.4) we know that

$$
g_{H}(-\gamma)=\left(1-g_{-}(-\gamma)\right)^{-1}
$$

By (3.4) and the Blackwell renewal theorem, we have, for $m>0$ and each $a>0$,

$$
H(-x+a)-H(-x) \rightarrow m^{-1} a(1-\alpha)
$$


Now we deal with $I(x)$. For any fixed positive integer $N$, by Fubini's theorem we have

$$
\begin{aligned}
I(x) & =\sum_{n=0}^{\infty} \int_{-\infty}^{0} l(x-y) \mathrm{P}\left(S_{n} \in \mathrm{d} y, \Lambda_{n}\right) \\
& =\int_{-\infty}^{0} l(x-y) H(\mathrm{~d} y) \\
& =\left(\int_{-N}^{0}+\int_{-\infty}^{-N}\right) l(x-y) H(\mathrm{~d} y) \\
& =: I_{1}(x)+I_{2}(x) .
\end{aligned}
$$

By $l \in \mathcal{L} d(\gamma)$ and the dominated convergence theorem,

$$
I_{1}(x) \sim l(x) \int_{-N}^{0} \mathrm{e}^{\gamma y} H(\mathrm{~d} y) .
$$

By (3.6), $l \in \mathcal{L} d(\gamma)$, Karamata's theorem, and (3.3), when $N$ is sufficiently large, for any $x>0$,

$$
\begin{aligned}
I_{2}(x) & =\sum_{j=N}^{\infty} \int_{-j-1}^{-j} l(x-y) H(\mathrm{~d} y) \\
& =\sum_{j=N}^{\infty} \int_{-1}^{0} l(x+j-z) \mathrm{d} H(z-j) \\
& \leq 2 \sum_{j=N}^{\infty} l(x+j) \int_{-1}^{0} \mathrm{e}^{\gamma z} \mathrm{~d} H(z-j) \\
& \leq 2 \sum_{j=N}^{\infty} l(x+j)(H(-j)-H(-j-1)) \\
& \leq 3 m^{-1}(1-\alpha) \sum_{j=N}^{\infty} l(x+j) \\
& \leq 4 m^{-1}(1-\alpha) \gamma\left(1-\mathrm{e}^{-\gamma}\right)^{-1} \sum_{j=N}^{\infty} \int_{j}^{j+1} l(x+y) \mathrm{d} y \\
& =4 m^{-1}(1-\alpha) \gamma\left(1-\mathrm{e}^{-\gamma}\right)^{-1} \int_{x+N}^{\infty} l(y) \mathrm{d} y \\
& \leq 5 m^{-1}(1-\alpha)\left(1-\mathrm{e}^{-\gamma}\right)^{-1} l(x+N) .
\end{aligned}
$$

By (3.5) and (3.7)-(3.9), first letting $x \rightarrow \infty$ and then letting $N \rightarrow \infty$, we know that (3.2) holds.

\section{Asymptotics for the moments of the first ascending ladder height}

In this section we will investigate the local and nonlocal asymptotics for the moments of the first ascending ladder height of a random walk for the heavy-tailed and light-tailed cases. 


\subsection{Heavy-tailed case}

Theorem 4.1. Assume that $\varphi \in \mathcal{L} d$. Then the following assertions hold.

(i) If $F^{I} \in \mathcal{L}_{\triangle_{T}}$ for some $0<T<\infty$ then

$$
\operatorname{E} \varphi\left(S_{\tau_{+}}\right) \mathbf{1}_{\left\{S_{\tau_{+}} \in x+\triangle_{T}\right\}} \sim(1-\alpha) m^{-1} \int_{x}^{x+T} \varphi(y) \bar{F}(y) \mathrm{d} y .
$$

(ii) If $F^{I} \in \mathcal{L}_{\triangle_{T_{1}}}$ for some $0<T_{1}<\infty$ and $\int_{0}^{\infty} \varphi(y) \bar{F}(y) \mathrm{d} y<\infty$, then

$$
\operatorname{E} \varphi\left(S_{\tau_{+}}\right) \mathbf{1}_{\left\{S_{\tau_{+}}>x\right\}} \sim(1-\alpha) m^{-1} \int_{x}^{\infty} \varphi(y) \bar{F}(y) \mathrm{d} y .
$$

Taking $\varphi \equiv 1$ in Theorem 4.1(i), we can obtain Lemma 3 of Asmussen et al. (2002), i.e.

$$
\mathrm{P}\left(S_{\tau_{+}} \in x+\triangle_{T}\right) \sim(1-\alpha) m^{-1} \int_{x}^{x+T} \bar{F}(y) \mathrm{d} y .
$$

Theorem 4.2. (i) If $F^{I} \in \mathcal{L}_{\triangle_{T}}$ for some $0<T<\infty, \varphi \in \mathcal{L} d$, and $\varphi$ satisfies (2.1) and (2.2), then

$$
\mathrm{E} \varphi\left(S_{\tau_{+}}-x\right) \mathbf{1}_{\left\{S_{\tau_{+}}>x\right\}} \sim(1-\alpha) m^{-1} \int_{0}^{\infty} \varphi(y) \bar{F}(y+x) \mathrm{d} y .
$$

(ii) If $F^{I} \in \mathcal{L}_{\triangle_{t}}$ for any $0<t<\infty$ and $\varphi$ is Riemann-Stieltjes integrable on every compact subset of $[0, \infty)$, then, for any $0<T<\infty$,

$$
\mathrm{E} \varphi\left(S_{\tau_{+}}-x\right) \mathbf{1}_{\left\{S_{\tau_{+}} \in x+\Delta_{T}\right\}} \sim(1-\alpha) m^{-1} \int_{0}^{T} \varphi(y) \bar{F}(y+x) \mathrm{d} y .
$$

Theorem 4.3. Assume that $\varphi \in \mathcal{L} d$. Then the following assertions hold.

(i) If $F^{I} \in \mathcal{L}$ and (2.1) is satisfied, then

$$
\mathrm{E} \varphi\left(x-S_{\tau_{+}-1}\right) \mathbf{1}_{\left\{S_{\tau_{+}}>x\right\}} \sim(1-\alpha) m^{-1} \int_{x}^{\infty} \varphi(y) \bar{F}(y) \mathrm{d} y .
$$

(ii) If $F \in \mathcal{L}_{\triangle_{T_{1}}}$ for some $0<T_{1}<\infty$ and $\int_{0}^{\infty} \varphi(y) F(\mathrm{~d} y)<\infty$, then

$$
\mathrm{E} \varphi\left(x-S_{\tau_{+}-1}\right) \mathbf{1}_{\left\{S_{\tau_{+}} \in x+\triangle_{T_{1}}\right\}} \sim(1-\alpha) m^{-1} \int_{x}^{\infty} \varphi(y) F\left(y+\triangle_{T_{1}}\right) \mathrm{d} y .
$$

Taking $\varphi \equiv 1$ in Theorem 4.3(i), we can obtain the following result of Theorem 10 of Borovkov (1976, Chapter 4):

$$
\mathrm{P}\left(S_{\tau_{+}}>x\right) \sim(1-\alpha) m^{-1} \overline{F^{I}}(x) .
$$

Proof of Theorem 4.1. (i) Taking $u=\bar{F}$ in Lemma 3.1, we obtain $U(x)=\overline{F^{I}}(x), U_{T}(x)=$ $F^{I}\left(x+\triangle_{T}\right)$, and $u_{T}(x)=F\left(x+\triangle_{T}\right)$. Since $F^{I} \in \mathcal{L}_{\triangle_{T}}$, then the conditions of Lemma 3.1 
are satisfied. It follows from $\varphi \in \mathcal{L} d$ that

$$
\begin{aligned}
\operatorname{E} \varphi\left(S_{\tau_{+}}\right) \mathbf{1}_{\left\{S_{\tau_{+}} \in x+\triangle_{T}\right\}} & =\sum_{n=1}^{\infty} \operatorname{E} \varphi\left(S_{n}\right) \mathbf{1}_{\left\{S_{\tau_{+}} \in x+\triangle_{T}, \tau_{+}=n\right\}} \\
& =\sum_{n=1}^{\infty} \int_{-\infty}^{0} \int_{x-y}^{x-y+T} \varphi(z+y) F(\mathrm{~d} z) \mathrm{P}\left(S_{n-1} \in \mathrm{d} y, \Lambda_{n-1}\right) \\
& =\sum_{n=0}^{\infty} \int_{-\infty}^{0} \int_{0}^{T} \varphi(x+t) F(x-y+\mathrm{d} t) \mathrm{P}\left(S_{n} \in \mathrm{d} y, \Lambda_{n}\right) \\
& \sim \varphi(x) \sum_{n=0}^{\infty} \int_{-\infty}^{0} F\left(x-y+\triangle_{T}\right) \mathrm{P}\left(S_{n} \in \mathrm{d} y, \Lambda_{n}\right) .
\end{aligned}
$$

By Lemma 3.1 and $F^{I} \in \mathcal{L}_{\triangle_{T}}$, we obtain

$$
\begin{aligned}
\sum_{n=0}^{\infty} \int_{-\infty}^{0} F\left(x-y+\triangle_{T}\right) \mathrm{P}\left(S_{n} \in \mathrm{d} y, \Lambda_{n}\right) & =\sum_{n=0}^{\infty} \mathrm{E} u_{T}\left(x-S_{n}\right) \mathbf{1}_{\Lambda_{n}} \\
& \sim(1-\alpha) m^{-1} F^{I}\left(x+\triangle_{T}\right) .
\end{aligned}
$$

So, by (4.7), (4.8), and $\varphi \in \mathcal{L} d$, we know that (4.1) holds.

(ii) It follows from Lemma 3.1 that (4.8) still holds for $0<T_{1}<\infty$. So, by Fubini's theorem, $\varphi \in \mathcal{L} d$, and (4.8), we have

$$
\begin{aligned}
\mathrm{E} \varphi\left(S_{\tau_{+}}\right) \mathbf{1}_{\left\{S_{\tau_{+}}>x\right\}} & =\sum_{n=1}^{\infty} \mathrm{E} \varphi\left(S_{n}\right) \mathbf{1}_{\left\{S_{n}>x, \tau_{+}=n\right\}} \\
& =\sum_{n=0}^{\infty} \int_{-\infty}^{0} \int_{x-y}^{\infty} \varphi(z+y) F(\mathrm{~d} z) \mathrm{P}\left(S_{n} \in \mathrm{d} y, \Lambda_{n}\right) \\
& =\sum_{n=0}^{\infty} \int_{-\infty}^{0} \sum_{k=1}^{\infty} \int_{x-y+(k-1) T_{1}}^{x-y+k T_{1}} \varphi(z+y) F(\mathrm{~d} z) \mathrm{P}\left(S_{n} \in \mathrm{d} y, \Lambda_{n}\right) \\
& \sim \sum_{k=1}^{\infty} \varphi\left(x+k T_{1}\right) \sum_{n=0}^{\infty} \int_{-\infty}^{0} F\left(x-y+(k-1) T_{1}+\triangle_{T_{1}}\right) \mathrm{P}\left(S_{n} \in \mathrm{d} y, \Lambda_{n}\right) \\
& \sim(1-\alpha) m^{-1} \sum_{k=1}^{\infty} \varphi\left(x+k T_{1}\right) F^{I}\left(x+(k-1) T_{1}+\triangle_{T_{1}}\right) \\
& \sim(1-\alpha) m^{-1} \sum_{k=1}^{\infty} \int_{x+(k-1) T_{1}}^{x+k T_{1}} \varphi(y) \bar{F}(y) \mathrm{d} y \\
& =(1-\alpha) m^{-1} \int_{x}^{\infty} \varphi(y) \bar{F}(y) \mathrm{d} y,
\end{aligned}
$$

that is, (4.2) holds. 
Proof of Theorem 4.2. (i) For any fixed positive integer $N$,

$$
\begin{aligned}
\mathrm{E} \varphi\left(S_{\tau_{+}}-x\right) \mathbf{1}_{\left\{S_{\tau_{+}}>x\right\}} \\
\quad=\sum_{n=1}^{\infty} \mathrm{E} \varphi\left(S_{n}-x\right) \mathbf{1}_{\left\{S_{n}>x, \tau_{+}=n\right\}} \\
\quad=\sum_{n=0}^{\infty} \int_{-\infty}^{0}\left(\sum_{k=1}^{N}+\sum_{k=N+1}^{\infty}\right) \int_{x-y+(k-1) T}^{x-y+k T} \varphi(z+y-x) F(\mathrm{~d} z) \mathrm{P}\left(S_{n} \in \mathrm{d} y, \Lambda_{n}\right) \\
=: E_{1}(x)+E_{2}(x) .
\end{aligned}
$$

We first deal with $E_{1}(x)$. Since $F^{I} \in \mathcal{L}_{\triangle_{T}}$ and $\varphi \in \mathcal{L} d$, by the proof of Theorem 4.1(i) we find that (4.8) still holds. So, by Fubini's theorem, (2.2), (4.8), and $F^{I} \in \mathcal{L}_{\triangle_{T}}$, we obtain, for sufficiently large $x$,

$$
\begin{aligned}
E_{1}(x) & \leq \bar{\varphi}_{N T} \sum_{k=1}^{N} \sum_{n=0}^{\infty} \int_{-\infty}^{0} F\left(x-y+(k-1) T+\triangle_{T}\right) \mathrm{P}\left(S_{n} \in \mathrm{d} y, \Lambda_{n}\right) \\
& \sim \bar{\varphi}_{N T}(1-\alpha) m^{-1}\left(\overline{F^{I}}(x)-\overline{F^{I}}(x+N T)\right) \\
& =o(1) \overline{F^{I}}(x) .
\end{aligned}
$$

Now consider $E_{2}(x)$. Again, by $\varphi \in \mathcal{L} d$, (4.8), and Fubini's theorem, for any $0<\varepsilon<1$, first letting $N$ be sufficiently large and then letting $x$ be sufficiently large, we obtain

$$
\begin{aligned}
E_{2}(x) & \leq(1+\varepsilon) \sum_{n=0}^{\infty} \int_{-\infty}^{0} \sum_{k=N+1}^{\infty} \varphi(k T) F\left(x-y+(k-1) T+\triangle_{T}\right) \mathrm{P}\left(S_{n} \in \mathrm{d} y, \Lambda_{n}\right) \\
& =(1+\varepsilon) \sum_{k=N+1}^{\infty} \varphi(k T) \sum_{n=0}^{\infty} \int_{-\infty}^{0} F\left(x-y+(k-1) T+\triangle_{T}\right) \mathrm{P}\left(S_{n} \in \mathrm{d} y, \Lambda_{n}\right) \\
& \leq(1+\varepsilon)^{2}(1-\alpha) m^{-1} \sum_{k=N+1}^{\infty} \varphi(k T) F^{I}\left(x+(k-1) T+\triangle_{T}\right) \\
& \leq(1+\varepsilon)^{3}(1-\alpha) m^{-1} \sum_{k=N+1}^{\infty} \int_{x+(k-1) T}^{x+k T} \varphi(y-x) \bar{F}(y) \mathrm{d} y \\
& =(1+\varepsilon)^{3}(1-\alpha) m^{-1}\left(\int_{x}^{\infty}-\int_{x}^{x+N T}\right) \varphi(y-x) \bar{F}(y) \mathrm{d} y \\
& =:(1+\varepsilon)^{3}(1-\alpha) m^{-1}\left(E_{21}(x)-E_{22}(x)\right) .
\end{aligned}
$$

By (2.2) and $F^{I} \in \mathcal{L}_{\triangle_{T}}$,

$$
E_{22}(x) \leq \bar{\varphi}_{N T}\left(\overline{F^{I}}(x)-\overline{F^{I}}(x+N T)\right)=o(1) \overline{F^{I}}(x) .
$$

Since $\varphi \in \mathcal{L} d$, by (2.1), there exists a positive constant $C_{1}=C_{1}(N, T)$ such that

$$
E_{21}(x) \geq C_{1} \overline{F^{I}}(x) .
$$


Hence, by (4.10)-(4.12), we obtain

$$
\begin{aligned}
E_{2}(x) & \lesssim(1-\alpha) m^{-1} \int_{x}^{\infty} \varphi(y-x) \bar{F}(y) \mathrm{d} y \\
& =(1-\alpha) m^{-1} \int_{0}^{\infty} \varphi(y) \bar{F}(y+x) \mathrm{d} y .
\end{aligned}
$$

Similarly, we can prove that

$$
(1-\alpha) m^{-1} \int_{0}^{\infty} \varphi(y) \bar{F}(y+x) \mathrm{d} y \lesssim E_{2}(x) .
$$

So, by (4.9), (4.13), and (4.14), we know that (4.3) holds.

(ii) For any positive integer $l$, let

$$
\bar{\varphi}_{l, k}=\sup _{y \in\left(l^{-1}(k-1) T, l^{-1} k T\right]} \varphi(y), \quad k=1,2, \ldots, l .
$$

Since $\varphi$ is Riemann-Stieltjes integrable, then, for any $0<\varepsilon<1$, there exists a positive constant $l_{0}$ (independent of $x$ ) such that, when $l \geq l_{0}$,

$$
\begin{aligned}
& \sum_{k=1}^{l} \bar{\varphi}_{l, k}\left(\overline{F^{I}}\left(x+l^{-1}(k-1) T\right)-\overline{F^{I}}\left(x+l^{-1} k T\right)\right) \\
& \quad \leq(1+\varepsilon) \sum_{k=1}^{l} \int_{x+l^{-1}(k-1) T}^{x+l^{-1} k T} \varphi(y-x) \bar{F}(y) \mathrm{d} y \\
& \quad=(1+\varepsilon) \int_{x}^{x+T} \varphi(y-x) \bar{F}(y) \mathrm{d} y .
\end{aligned}
$$

For the above given $\varepsilon$ and $l \geq l_{0}$, since $F^{I} \in \mathcal{L}_{\triangle_{t}}$ for any $t>0$, by (4.8) we know that there exists an $x_{0}>0$ such that, when $x \geq x_{0}$,

$$
\begin{aligned}
\mathrm{E} \varphi\left(S_{\tau_{+}}-x\right) \mathbf{1}_{\left\{S_{\tau_{+}} \in x+\triangle_{T}\right\}} \\
\quad=\sum_{n=1}^{\infty} \mathrm{E} \varphi\left(S_{n}-x\right) \mathbf{1}_{\left\{S_{\tau_{+}} \in x+\triangle_{T}, \tau_{+}=n\right\}} \\
\quad=\sum_{n=0}^{\infty} \int_{-\infty}^{0} \sum_{k=1}^{l} \int_{x-y+l^{-1}(k-1) T}^{x-y+l^{-1} k T} \varphi(z+y-x) F(\mathrm{~d} z) \mathrm{P}\left(S_{n} \in \mathrm{d} y, \Lambda_{n}\right) \\
\quad \leq \sum_{k=1}^{l} \bar{\varphi}_{l, k} \sum_{n=0}^{\infty} \int_{-\infty}^{0}\left(\bar{F}\left(x-y+l^{-1}(k-1) T\right)-\bar{F}\left(x-y+l^{-1} k T\right)\right) \mathrm{P}\left(S_{n} \in \mathrm{d} y, \Lambda_{n}\right) \\
\quad \leq(1+\varepsilon)(1-\alpha) m^{-1} \sum_{k=1}^{l} \bar{\varphi}_{l, k}\left(\bar{F}^{I}\left(x+l^{-1}(k-1) T\right)-\bar{F}^{I}\left(x+l^{-1} k T\right)\right) .
\end{aligned}
$$

Combining the above with (4.16), we obtain

$$
\mathrm{E} \varphi\left(S_{\tau_{+}}-x\right) \mathbf{1}_{\left\{S_{\tau_{+}} \in x+\triangle_{T}\right\}} \lesssim(1-\alpha) m^{-1} \int_{0}^{T} \varphi(y) \bar{F}(x+y) \mathrm{d} y .
$$


Similarly, we can prove that

$$
(1-\alpha) m^{-1} \int_{0}^{T} \varphi(y) \bar{F}(x+y) \mathrm{d} y \lesssim \mathrm{E} \varphi\left(S_{\tau_{+}}-x\right) \mathbf{1}_{\left\{S_{\tau_{+}} \in x+\triangle_{T}\right\}} .
$$

So (4.4) holds.

Proof of Theorem 4.3. (i) Taking $T=\infty, u_{1}=\varphi$, and $u_{2}=\bar{F}$ in Lemma 3.1, then $u_{1} \in \mathcal{L} d$ and $u_{2}$ is nonincreasing. It follows from (2.1) that $U(\infty)=u(\infty)=0$ and $U_{\infty}(x)=U(x)=\int_{x}^{\infty} \varphi(y) \bar{F}(y) \mathrm{d} y, x \in(-\infty, \infty)$. We will prove that $U_{\infty} \in \mathcal{L} d$.

By (2.1) we know that there exists a positive constant $C$ such that, for sufficiently large $x$, $\inf _{y \geq x} \varphi(y) \geq C \varphi(x)$. Hence,

$$
U_{\infty}(x)=\int_{x}^{\infty} \varphi(y) \bar{F}(y) \mathrm{d} y \geq C \varphi(x) \overline{F^{I}}(x) .
$$

The following fact is well known: $F^{I} \in \mathcal{L}$ if and only if

$$
\bar{F}(x)=o(1) \overline{F^{I}}(x) .
$$

Now, by $\varphi \in \mathcal{L} d,(4.17)$, and (4.18),

$$
\begin{aligned}
0 & \leq U_{\infty}(x)-U_{\infty}(x+1) \\
& =\int_{x}^{x+1} \varphi(y) \bar{F}(y) \mathrm{d} y \\
& \sim \varphi(x) \int_{x}^{x+1} \bar{F}(y) \mathrm{d} y \\
& \leq \varphi(x) \bar{F}(x) \\
& =o(1) U_{\infty}(x)
\end{aligned}
$$

that is, $U_{\infty} \in \mathcal{L} d$. Therefore, by Lemma 3.1 and Fubini's theorem, we obtain

$$
\begin{aligned}
\mathrm{E} \varphi\left(x-S_{\tau_{+}-1}\right) \mathbf{1}_{\left\{S_{\tau_{+}}>x\right\}} & =\sum_{n=1}^{\infty} \mathrm{E} \varphi\left(x-S_{n-1}\right) \mathbf{1}_{\left\{S_{n}>x, \tau_{+}=n\right\}} \\
& =\sum_{n=1}^{\infty} \int_{-\infty}^{0} \varphi(x-y) \bar{F}(x-y) \mathrm{P}\left(S_{n-1} \in \mathrm{d} y, \Lambda_{n-1}\right) \\
& =\sum_{n=0}^{\infty} \mathrm{E} u_{\infty}\left(x-S_{n}\right) \mathbf{1}_{\Lambda_{n}} \\
& \sim(1-\alpha) m^{-1} \int_{x}^{\infty} \varphi(y) \bar{F}(y) \mathrm{d} y .
\end{aligned}
$$

(ii) Taking $T=\infty, u_{1}(x)=\varphi(x) F\left(x+\triangle_{T_{1}}\right)$, and $u_{2} \equiv 1$ in Lemma 3.1, then $u=u_{1} \in \mathcal{L} d$. Combining this with $\int_{0}^{\infty} \varphi(y) F(\mathrm{~d} y)<\infty$, we have $u(\infty)=0, U_{\infty}(x)=U(x)=$ $\int_{x}^{\infty} \varphi(y) F\left(y+\triangle_{T_{1}}\right) \mathrm{d} y, x \in(-\infty, \infty)$, and $U(\infty)=0$. In fact, for sufficiently large $x$,

$$
\begin{aligned}
U(x) & =\int_{x}^{\infty} u(y) \mathrm{d} y \\
& =\sum_{k=0}^{\infty} \int_{x+k T_{1}}^{x+(k+1) T_{1}} u(y) \mathrm{d} y
\end{aligned}
$$




$$
\begin{aligned}
& \sim T_{1} \sum_{k=0}^{\infty} \varphi\left(x+k T_{1}\right) \int_{x+k T_{1}}^{x+(k+1) T_{1}} F(\mathrm{~d} y) \\
& \sim T_{1} \sum_{k=0}^{\infty} \int_{x+k T_{1}}^{x+(k+1) T_{1}} \varphi(y) F(\mathrm{~d} y) \\
& =T_{1} \int_{x}^{\infty} \varphi(y) F(\mathrm{~d} y) \\
& <\infty .
\end{aligned}
$$

By $u \in \mathcal{L} d, \int_{0}^{\infty} \varphi(y) F(\mathrm{~d} y)<\infty$, and Karamata's theorem, we obtain

$$
U_{\infty}(x)-U_{\infty}(x+1)=\int_{x}^{x+1} u(y) \mathrm{d} y \sim u(x)=o(1) U_{\infty}(x) .
$$

Thus, $U_{\infty} \in \mathcal{L} d$. Again, using Lemma 3.1, we know that (4.6) holds.

\subsection{Light-tailed case}

Theorem 4.4. Assume that $\varphi \in \mathcal{L} d$ and that $\int_{0}^{\infty} \varphi(y) \bar{F}(y) \mathrm{d} y<\infty$. For some $\gamma>0$, let $F \in \mathcal{L}(\gamma)$ and $g_{F}(-\gamma)<1$. Then

$$
\mathrm{E} \varphi\left(x-S_{\tau_{+}-1}\right) \mathbf{1}_{\left\{S_{\tau_{+}}>x\right\}} \sim\left(1-g_{-}(-\gamma)\right)^{-1} \varphi(x) \bar{F}(x) .
$$

Letting $\varphi \equiv 1$ in (4.19), we can obtain another result of Theorem 10 of Borovkov (1976, Chapter 4), i.e.

$$
\begin{aligned}
\mathrm{P}\left(S_{\tau_{+}}>x\right) & =\sum_{n=0}^{\infty} \int_{-\infty}^{0} \bar{F}(x-y) \mathrm{P}\left(S_{n} \in \mathrm{d} y, \Lambda_{n}\right) \\
& \sim\left(1-g_{-}(-\gamma)\right)^{-1} \bar{F}(x) .
\end{aligned}
$$

By (4.20) and $F \in \mathcal{L}(\gamma), \gamma>0$, we can obtain, for any $0<T<\infty$,

$$
\begin{aligned}
\mathrm{P}\left(S_{\tau_{+}} \in x+\triangle_{T}\right) & =\sum_{n=0}^{\infty} \int_{-\infty}^{0} F\left(x-y+\triangle_{T}\right) \mathrm{P}\left(S_{n} \in \mathrm{d} y, \Lambda_{n}\right) \\
& \sim\left(1-g_{-}(-\gamma)\right)^{-1}\left(1-\mathrm{e}^{-\gamma T}\right) \bar{F}(x) .
\end{aligned}
$$

Theorem 4.5. Assume that the conditions of Theorem 4.4 hold and that $\varphi$ is Riemann-Stieltjes integrable on every compact subset of $[0, \infty)$. Then

$$
\operatorname{E} \varphi\left(S_{\tau_{+}}-x\right) \mathbf{1}_{\left\{S_{\tau_{+}}>x\right\}} \sim\left(1-g_{-}(-\gamma)\right)^{-1} \int_{x}^{\infty} \varphi(y-x) F(\mathrm{~d} y)
$$

and, for any $0<T<\infty$,

$$
\mathrm{E} \varphi\left(S_{\tau_{+}}-x\right) \mathbf{1}_{\left\{S_{\tau_{+}} \in x+\Delta_{T}\right\}} \sim\left(1-g_{-}(-\gamma)\right)^{-1} \int_{x}^{x+T} \varphi(y-x) F(\mathrm{~d} y) .
$$

Theorem 4.6. Under the conditions of Theorem 4.4, we have, for any $0<T \leq \infty$,

$$
\mathrm{E} \varphi\left(S_{\tau_{+}}\right) \mathbf{1}_{\left\{S_{\tau_{+}} \in x+\triangle_{T}\right\}} \sim\left(1-g_{-}(-\gamma)\right)^{-1} \int_{x}^{x+T} \varphi(y) F(\mathrm{~d} y)
$$

and $\int_{0}^{\infty} \varphi(y) F(\mathrm{~d} y)<\infty$. 
Proof of Theorem 4.4.. Equation (4.19) can be obtained by taking $l(x)=\varphi(x) \bar{F}(x)$ in Lemma 3.2.

Proof of Theorem 4.5.. For any $\varepsilon>0$ and a fixed positive integer $N$, we obtain

$$
\begin{aligned}
\operatorname{E} \varphi\left(S_{\tau_{+}}-x\right) \mathbf{1}_{\left\{S_{\tau_{+}}>x\right\}} \\
\quad=\sum_{n=0}^{\infty} \int_{-\infty}^{0}\left(\sum_{k=1}^{N}+\sum_{k=N+1}^{\infty}\right) \int_{x-y+(k-1) \varepsilon}^{x-y+k \varepsilon} \varphi(z+y-x) F(\mathrm{~d} z) \mathrm{P}\left(S_{n} \in \mathrm{d} y, \Lambda_{n}\right) \\
\quad=: E_{1}(x)+E_{2}(x) .
\end{aligned}
$$

We first deal with $E_{2}(x)$. By $\varphi \in \mathcal{L} d$, for any $0<\delta<1$ and sufficiently large $N$,

$$
\begin{aligned}
E_{2}(x) & \leq \sum_{k=N+1}^{\infty} \varphi((k-1) \varepsilon) \sum_{n=0}^{\infty} \int_{-\infty}^{0} F\left(x-y+(k-1) \varepsilon+\triangle_{\varepsilon}\right) \mathrm{P}\left(S_{n} \in \mathrm{d} y, \Lambda_{n}\right) \\
& \leq(1+\delta)\left(1-g_{-}(-\gamma)\right)^{-1} \sum_{k=N+1}^{\infty} \varphi((k-1) \varepsilon)(\bar{F}(x+(k-1) \varepsilon)-\bar{F}(x+k \varepsilon)) \\
& \leq(1+\delta)^{2}\left(1-g_{-}(-\gamma)\right)^{-1} \int_{x+N \varepsilon}^{\infty} \varphi(y-x) F(\mathrm{~d} y) .
\end{aligned}
$$

Now consider $E_{1}(x)$. Let $\bar{\varphi}_{l, k}=\sup _{y \in\left(l^{-1}(k-1) N \varepsilon, l^{-1} k N \varepsilon\right]} \varphi(y)$, where $l$ is a positive integer and $k=1,2, \ldots, l$. For the above fixed $\varepsilon$ and $N$, by Fubini's theorem, (4.20), and the fact that $\varphi$ is Riemann-Stieltjes integrable, we know that, when $x$ and $l$ are sufficiently large,

$$
\begin{aligned}
E_{1}(x) & =\sum_{n=0}^{\infty} \int_{-\infty}^{0} \int_{x-y}^{x-y+N \varepsilon} \varphi(z+y-x) F(\mathrm{~d} z) \mathrm{P}\left(S_{n} \in \mathrm{d} y, \Lambda_{n}\right) \\
& =\sum_{n=0}^{\infty} \int_{-\infty}^{0} \sum_{k=1}^{l} \int_{x-y+l^{-1}(k-1) N \varepsilon}^{x-y+l^{-1} k N \varepsilon} \varphi(z+y-x) F(\mathrm{~d} z) \mathrm{P}\left(S_{n} \in \mathrm{d} y, \Lambda_{n}\right) \\
& \leq \sum_{k=1}^{l} \bar{\varphi}_{l, k} \sum_{n=0}^{\infty} \int_{-\infty}^{0} F\left(x-y+l^{-1}(k-1) N \varepsilon+\triangle_{l^{-1} N \varepsilon}\right) \mathrm{P}\left(S_{n} \in \mathrm{d} y, \Lambda_{n}\right) \\
& \leq(1+\delta)\left(1-g_{-}(-\gamma)\right)^{-1} \sum_{k=1}^{l} \bar{\varphi}_{l, k}\left(\bar{F}\left(x+l^{-1}(k-1) N \varepsilon\right)-\bar{F}\left(x+l^{-1} k N \varepsilon\right)\right) \\
& \leq(1+\delta)^{2}\left(1-g_{-}(-\gamma)\right)^{-1} \sum_{k=1}^{l} \int_{x+l^{-1}(k-1) N \varepsilon}^{x+l^{-1} k N \varepsilon} \varphi(y-x) F(\mathrm{~d} y) \\
& =(1+\delta)^{2}\left(1-g_{-}(-\gamma)\right)^{-1} \int_{x}^{x+N \varepsilon} \varphi(y-x) F(\mathrm{~d} y) .
\end{aligned}
$$

Hence, for sufficiently large $x$,

$$
\mathrm{E} \varphi\left(S_{\tau_{+}}-x\right) \mathbf{1}_{\left\{S_{\tau_{+}}>x\right\}} \leq(1+\delta)^{2}\left(1-g_{-}(-\gamma)\right)^{-1} \int_{x}^{\infty} \varphi(y-x) F(\mathrm{~d} y) .
$$


Similarly, we can obtain

$$
\mathrm{E} \varphi\left(S_{\tau_{+}}-x\right) \mathbf{1}_{\left\{S_{\tau_{+}}>x\right\}} \geq(1-\delta)^{2}\left(1-g_{-}(-\gamma)\right)^{-1} \int_{x}^{\infty} \varphi(y-x) F(\mathrm{~d} y) .
$$

It follows from (4.27) and (4.28) that (4.22) holds.

The proof of (4.23) is similar to that of (4.22) and, thus, we omit the details.

Proof of Theorem 4.6. We first give a proof for the case $0<T<\infty$. Вy $\varphi \in \mathcal{L} d$, $F \in \mathcal{L}(\gamma)$, and (4.21),

$$
\begin{aligned}
\mathrm{E} \varphi\left(S_{\tau_{+}}\right) \mathbf{1}_{\left\{S_{\tau_{+}} \in x+\Delta_{T}\right\}} & =\sum_{n=1}^{\infty} \mathrm{E} \varphi\left(S_{n}\right) \mathbf{1}_{\left\{S_{\tau_{+}} \in x+\triangle_{T}, \tau_{+}=n\right\}} \\
& =\sum_{n=1}^{\infty} \mathrm{E} \varphi\left(X_{n}+S_{n-1}\right) \mathbf{1}_{\left\{x-S_{n-1}<X_{n} \leq x+T-S_{n-1}, \tau_{+}=n\right\}} \\
& =\sum_{n=0}^{\infty} \int_{-\infty}^{0} \int_{x-y}^{x-y+T} \varphi(y+z) F(\mathrm{~d} z) \mathrm{P}\left(S_{n} \in \mathrm{d} y, \Lambda_{n}\right) \\
& \sim \varphi(x) \sum_{n=0}^{\infty} \int_{-\infty}^{0} F\left(x-y+\triangle_{T}\right) \mathrm{P}\left(S_{n} \in \mathrm{d} y, \Lambda_{n}\right) \\
& \sim\left(1-g_{-}(-\gamma)\right)^{-1}\left(1-\mathrm{e}^{-\gamma T}\right) \varphi(x) \bar{F}(x) \\
& \sim\left(1-g_{-}(-\gamma)\right)^{-1} \int_{x}^{x+T} \varphi(y) F(\mathrm{~d} y)
\end{aligned}
$$

i.e. (4.24) holds for $0<T<\infty$.

Before giving a proof for the case $T=\infty$, we need to prove that $\int_{0}^{\infty} \varphi(y) F(\mathrm{~d} y)<\infty$. By $F \in \mathcal{L}(\gamma)$, it is easy to show that, for any positive constant $C$,

$$
\int_{x}^{x+C} \varphi(y) F(\mathrm{~d} y) \sim\left(1-\mathrm{e}^{-\gamma C}\right)\left(\int_{0}^{C} \mathrm{e}^{-\gamma y} \mathrm{~d} y\right)^{-1} \int_{x}^{x+C} \varphi(y) \bar{F}(y) \mathrm{d} y .
$$

Hence,

$$
\int_{0}^{\infty} \varphi(y) \bar{F}(y) \mathrm{d} y<\infty \quad \Longleftrightarrow \int_{0}^{\infty} \varphi(y) F(\mathrm{~d} y)<\infty .
$$

For the case $T=\infty$, by Fubini's theorem, $\varphi \in \mathcal{L} d$, and the above proof for the case $0<T<\infty$, we have, for any $0<T_{1}<\infty$,

$$
\begin{aligned}
\operatorname{E} \varphi\left(S_{\tau_{+}}\right) \mathbf{1}_{\left\{S_{\tau_{+}}>x\right\}} & =\sum_{n=1}^{\infty} \operatorname{E} \varphi\left(S_{n}\right) \mathbf{1}_{\left\{S_{n}>x, \tau_{+}=n\right\}} \\
& =\sum_{k=1}^{\infty} \sum_{n=0}^{\infty} \int_{-\infty}^{0} \int_{x-y+(k-1) T_{1}}^{x-y+k T_{1}} \varphi(z+y) F(\mathrm{~d} z) \mathrm{P}\left(S_{n} \in \mathrm{d} y, \Lambda_{n}\right) \\
& \sim\left(1-g_{-}(-\gamma)\right)^{-1} \sum_{k=1}^{\infty} \int_{x+(k-1) T_{1}}^{x+k T_{1}} \varphi(y) F(\mathrm{~d} y) \\
& =\left(1-g_{-}(-\gamma)\right)^{-1} \int_{x}^{\infty} \varphi(y) F(\mathrm{~d} y),
\end{aligned}
$$

i.e. (4.24) holds for $T=\infty$. 
Remark 4.1. If $\varphi$ is nondecreasing, using integration by parts, all the above results can also be verified. Combining this with Theorems 5.1 and 5.2, below, different proofs can be given for Theorems 3.2 and 3.4 of Cheng et al. (2002).

\section{On the solutions of renewal equations}

In this section we will give some equivalent conditions and sufficient conditions for the asymptotics of the solutions to some renewal equations. For this, we will introduce some notions and notation.

We consider the renewal equation $Z=z+q W * Z$, i.e.

$$
Z(x)=z(x)+q \int_{0}^{x} Z(x-y) W(\mathrm{~d} y), \quad x \geq 0,
$$

where $W$ is a proper distribution on $[0, \infty), z(x) \geq 0$ is a known and locally bounded function on $[0, \infty)$, and $q>0$ is a known constant. Throughout this section, we suppose that $0<q<1$. In this case, $Z=(1-q)^{-1} U_{0} * z$, i.e.

$$
Z(x)=(1-q)^{-1} \int_{0}^{x} z(x-y) U_{0}(\mathrm{~d} y),
$$

where

$$
U_{0}(x)=(1-q) \sum_{n=0}^{\infty} q^{n} W^{* n}(x), \quad x \geq 0 .
$$

Since in most cases it is not easy to calculate (5.2), attention is paid to asymptotics of the solution $Z(x)$. Asymptotics of $Z(x)$ have a close relation with the properties of $z(x)$. There are some existing results. For $z=W$, see Embrechts et al. (1979), Embrechts and Goldie (1982), Cline (1987), among others. For the case in which $z$ is a proper distribution, see Cai and Garrido (2002) and Cai and Tang (2004). For the case in which $z$ is a subexponential density, see Asmussen (1998) and Asmussen et al. (2003). Recently, Yin and Zhao (2006) investigated the case in which $(z(0))^{-1} z(x)$ is a tail distribution and obtained some new results. For the purpose of this paper, we will discuss asymptotics of $Z(x)$ for the new case

$$
z(x) \sim c \bar{L}(x)
$$

where $c$ is a positive constant and $L$ is a proper distribution on $[0, \infty)$. The results will be given for the cases in which $W$ is heavy tailed and light tailed.

\subsection{Heavy-tailed case}

Theorem 5.1. For the renewal equation (5.1), assume that (5.4) is satisfied.

Case 1. Assume that $\overline{U_{0}}(x) \approx \bar{L}(x), L \in \mathcal{L}$, and $U_{0} \in \mathcal{L}$. Then the following assertions are equivalent:

$$
\text { (i) } L \in 8 ; \quad \text { (ii) } W \in s ; \quad \text { (iii) } Z(x) \sim c(1-q)^{-1} \bar{L}(x) \text {. }
$$

Case 2. Assume that $\overline{U_{0}}(x)=o(1) \bar{L}(x)$ and $L \in$ \&. Then (iii) still holds.

Proof. Case 1. We first show that (i) implies (ii) and (iii). By $L \in \&, \overline{U_{0}}(x) \approx \bar{L}(x)$, and $U_{0} \in \mathcal{L}$, we know that $U_{0} \in \delta$. Hence, $W \in \delta$ by (5.3) and Corollary 3 of Embrechts $e t$ al. (1979). Now we prove that (iii) holds. For any fixed positive constant $N$ and any $x \geq 2 N$, 
by (5.2),

$$
\begin{aligned}
Z(x) & =(1-q)^{-1}\left(\int_{0}^{N}+\int_{N}^{x-N}+\int_{x-N}^{x}\right) z(x-y) U_{0}(\mathrm{~d} y) \\
& =: Z_{1}(x)+Z_{2}(x)+Z_{3}(x) .
\end{aligned}
$$

By (5.4), $L \in \mathcal{L}$, and the dominated convergence theorem,

$$
Z_{1}(x) \sim c(1-q)^{-1} \bar{L}(x) U_{0}(N) .
$$

Again, by (5.4), since $U_{0} \in \&$ and $\overline{U_{0}}(x) \approx \bar{L}(x)$, it is easy to show that

$$
\limsup _{N \rightarrow \infty} \lim \sup \frac{Z_{2}(x)}{\bar{L}(x)}=\limsup _{N \rightarrow \infty} \lim \sup \int_{N}^{x-N} \frac{\overline{U_{0}}(x-y)}{\overline{U_{0}}(x)} U_{0}(\mathrm{~d} y)=0 .
$$

For the above given $N$, since $\bar{z}_{N} \equiv \sup _{y \in[0, N]} z(y)<\infty$, by $U_{0} \in \mathcal{L}$ and $\overline{U_{0}}(x) \approx \bar{L}(x)$, we obtain

$$
Z_{3}(x) \leq(1-q)^{-1} \underline{z}_{N}\left(\overline{U_{0}}(x-N)-\overline{U_{0}}(x)\right)=o(1) \bar{L}(x) .
$$

Hence, (iii) holds by (5.5)-(5.8).

We will prove that (iii) implies (i). It follows from $L \in \mathcal{L}, U_{0} \in \mathcal{L}$, and $\overline{U_{0}}(x) \approx \bar{L}(x)$ that (5.6) and (5.8) still hold. Hence, (iii) tells us that the second limitation of (5.7) holds. Combining this with $U_{0} \in \mathcal{L}$, we know that $U_{0} \in \S$. So, $L \in \&$ follows from $L \in \mathcal{L}$, $\overline{U_{0}}(x) \approx \bar{L}(x)$, and $U_{0} \in \delta$.

Finally, we prove that (ii) implies (iii). By $W \in \&$ and Theorem 1 of Veraverbeke (1977), we know that $U_{0} \in \&$. Hence, $L \in \&$, i.e. (i) holds. By the above implication of (iii) by (i), (iii) holds.

Case 2. It is obvious that (5.6) and (5.8) still hold. Using integration by parts,

$$
\int_{N}^{x-N} \bar{L}(x-y) U_{0}(\mathrm{~d} y)=\bar{L}(x-N) \overline{U_{0}}(N)-\bar{L}(N) \overline{U_{0}}(x-N)+\int_{N}^{x-N} \overline{U_{0}}(x-y) L(\mathrm{~d} y) ;
$$

combining this with $\overline{U_{0}}(x)=o(1) \bar{L}(x)$ and $L \in \delta$, we know that the first limitation of (5.7) holds. Hence, (iii) holds. result.

If $L=W$ then we can cancel some conditions of Theorem 5.1 and obtain the following

Corollary 5.1. For the renewal equation (5.1), assume that (5.4) is satisfied for $L=W$. Then the following assertions are equivalent:

$$
\text { (i) } W \in s ; \quad \text { (ii) } W \in \mathcal{L} \text { and } Z(x) \sim c(1-q)^{-1} \bar{W}(x) \text {. }
$$

Proof. We only need to prove that (ii) implies (i). Since $W \in \mathcal{L}$, then (5.6) still holds for $L=W$. Combining this with (5.9), we obtain

$$
\limsup _{N \rightarrow \infty} \lim \sup \int_{N}^{x-N} \frac{\bar{W}(x-y)}{\bar{W}(x)} U_{0}(\mathrm{~d} y)=0 .
$$

Since $U_{0}(\mathrm{~d} y)=(1-q) \sum_{n=1}^{\infty} q^{n} W^{* n}(\mathrm{~d} y)$, it is obvious to show that

$$
\int_{N}^{x-N} \bar{W}(x-y) W(\mathrm{~d} y)=O(1) \int_{N}^{x-N} \bar{W}(x-y) U_{0}(\mathrm{~d} y) .
$$

Combining this with $W \in \mathcal{L}$, we know that $W \in \varsigma$. 


\subsection{Light-tailed case}

We primarily discuss the case in which $L=W$ in this subsection.

Theorem 5.2. For the renewal equation (5.1), assume that (5.4) is satisfied.

Case 1. Assume that $L=W \in \mathcal{L}(\gamma)$ for some $\gamma>0$ and that $g_{W}(-\gamma)<1$. Also, suppose that $z$ is continuous almost everywhere with respect to the Lebesgue measure. Then the following assertions are equivalent:

(i) $W \in \delta(\gamma)$;

(ii) $Z(x) \sim\left(c\left(1-q g_{W}(-\gamma)\right)^{-1}+I q\left(1-q g_{W}(-\gamma)\right)^{-2}\right) \bar{W}(x)$,

where $I=\gamma \int_{0}^{\infty} z(y) \mathrm{e}^{\gamma y} \mathrm{~d} y$.

Case 2. Assume that $\overline{U_{0}}(x)=o(1) \bar{L}(x)$ and that $L \in \delta(\gamma)$. Then

$$
Z(x) \sim c\left(1-q g_{W}(-\gamma)\right)^{-1} \bar{L}(x) .
$$

Proof. Case 1. We first prove that (i) implies (ii). It follows from (5.3), $W \in \delta(\gamma)$, and Theorem 1 of Veraverbeke (1977) that $U_{0} \in \delta(\gamma)$ and

$$
\overline{U_{0}}(x) \sim q(1-q)\left(1-q g_{W}(-\gamma)\right)^{-2} \bar{W}(x) .
$$

Since $U_{0} \in \delta(\gamma) \subset \mathcal{L}(\gamma)$, then there exists a nonnegative function $h(x)$ on $[0, \infty)$ such that

$$
h(x) \uparrow \infty, \quad \frac{h(x)}{x} \rightarrow 0, \quad \text { and } \quad \overline{U_{0}}(x-t) \sim \mathrm{e}^{\gamma t} \overline{U_{0}}(x)
$$

uniformly for $|t| \leq h(x)$,

$$
\begin{gathered}
U_{0} \in \delta(\gamma) \Longleftrightarrow U_{0} \in \mathcal{L}(\gamma), \quad g_{U_{0}}(-\gamma)<\infty, \\
\text { and } \int_{h(x)}^{x-h(x)} \overline{U_{0}}(x-y) U_{0}(\mathrm{~d} y)=o(1) \overline{U_{0}}(x) .
\end{gathered}
$$

Now we deal with $Z(x)$ using $h$ :

$$
\begin{aligned}
Z(x) & =(1-q)^{-1}\left(\int_{0}^{h(x)}+\int_{h(x)}^{x-h(x)}+\int_{x-h(x)}^{x}\right) z(x-y) U_{0}(\mathrm{~d} y) \\
& =: Z_{1}(x)+Z_{2}(x)+Z_{3}(x) .
\end{aligned}
$$

By $W \in \delta(\gamma) \subset \mathcal{L}(\gamma),(5.4)$, and the dominated convergence theorem,

$$
Z_{1}(x) \sim c(1-q)^{-1} g_{U_{0}}(-\gamma) \bar{W}(x)=c\left(1-q g_{W}(-\gamma)\right)^{-1} \bar{W}(x) .
$$

It follows from $U_{0} \in \delta(\gamma),(5.4)$, and (5.12) that

$$
\begin{aligned}
\int_{h(x)}^{x-h(x)} z(x-y) U_{0}(\mathrm{~d} y) & \sim c \int_{h(x)}^{x-h(x)} \bar{W}(x-y) U_{0}(\mathrm{~d} y) \\
& \sim c q^{-1}(1-q)^{-1}\left(1-q g_{W}(-\gamma)\right)^{2} \int_{h(x)}^{x-h(x)} \overline{U_{0}}(x-y) U_{0}(\mathrm{~d} y) \\
& =o(1) \overline{U_{0}}(x) \\
& =o(1) \bar{W}(x)
\end{aligned}
$$


For $Z_{3}(x)$, we use a different approach to that given in Theorem 5.1. We first show that $\mathrm{e}^{\gamma x} z(x)$ is directly Riemann integrable on $[0, \infty)$. For this, we need to prove that $\mathrm{e}^{\gamma x} \bar{W}(x)$ is directly Riemann integrable. Since $g_{W}(-\gamma)<1$, $\mathrm{e}^{\gamma x} \bar{W}(x)$ is integrable on $[0, \infty)$. So, for any $\delta>0$,

$$
\sum_{n=0}^{\infty} \mathrm{e}^{\gamma n \delta} \bar{W}((n-1) \delta)<\infty .
$$

Equation (5.16) and Proposition 4.1(ii) of Asmussen (2003, Chapter V) show that $\mathrm{e}^{\gamma x} \bar{W}(x)$ is directly Riemann integrable. Using (5.4) and the result that, for two nonnegative functions $f_{1}$ and $f_{2}$ on $[0, \infty)$ such that $f_{1}(x) \sim f_{2}(x), f_{1}$ is directly Riemann integrable is equivalent to $f_{2}$ being directly Riemann integrable, we can show that $\mathrm{e}^{\gamma x} z(x)$ is directly Riemann integrable.

For any $0<\varepsilon<1$, let

$$
\bar{z}_{\varepsilon}(x)=\sup _{y \in(x, x+\varepsilon]} z(y), \quad x \geq 0 .
$$

Hence, by $W \in \delta(\gamma), U_{0} \in \delta(\gamma) \subset \mathcal{L}(\gamma)$, and (5.12),

$$
\begin{aligned}
Z_{3}(x) & \leq(1-q)^{-1} \sum_{k=0}^{\varepsilon^{-1} h(x)} \bar{z}_{\varepsilon}(k \varepsilon) U_{0}(x-(k+1) \varepsilon, x-k \varepsilon] \\
& \sim(1-q)^{-1} \sum_{k=0}^{\varepsilon^{-1} h(x)} \bar{z}_{\varepsilon}(k \varepsilon)\left(\mathrm{e}^{\gamma(k+1) \varepsilon}-\mathrm{e}^{\gamma k \varepsilon}\right) \overline{U_{0}}(x) \\
& \sim q\left(1-q g_{W}(-\gamma)\right)^{-2} \sum_{k=0}^{\varepsilon^{-1} h(x)} \bar{z}_{\varepsilon}(k \varepsilon)\left(\mathrm{e}^{\gamma(k+1) \varepsilon}-\mathrm{e}^{\gamma k \varepsilon}\right) \bar{W}(x) .
\end{aligned}
$$

Hence,

$$
\lim _{\varepsilon \downarrow 0} \lim \sup \frac{Z_{3}(x)}{\bar{W}(x)} \leq q\left(1-q g_{W}(-\gamma)\right)^{-2} I .
$$

Similarly, writing $\underline{z}_{\varepsilon}(x)=\inf _{y \in(x, x+\varepsilon]} z(y), x \geq 0$, it can be proved that

$$
\lim _{\varepsilon \downarrow 0} \lim \inf \frac{Z_{3}(x)}{\bar{W}(x)} \geq q\left(1-q g_{W}(-\gamma)\right)^{-2} I .
$$

So (5.10) holds by (5.13)-(5.15), (5.17), and (5.18).

In the following we prove that (ii) implies (i). Since $W \in \mathcal{L}(\gamma)$, by (5.4), (5.14) still holds. It follows from (5.3), $W \in \mathcal{L}(\gamma)$, and Fatou's lemma that, for all $k \geq 1$,

$$
\begin{aligned}
\liminf \frac{(1-q)^{-1} U_{0}(x-k \varepsilon, x-(k-1) \varepsilon]}{q \bar{W}(x)} & \geq \sum_{i=1}^{\infty} \liminf \frac{q^{i} W^{* i}(x-k \varepsilon, x-(k-1) \varepsilon]}{q \bar{W}(x)} \\
& \geq\left(1-q g_{W}(-\gamma)\right)^{-2}\left(\mathrm{e}^{\gamma k \varepsilon}-\mathrm{e}^{\gamma(k-1) \varepsilon}\right) .
\end{aligned}
$$

Hence, by the fact that $\mathrm{e}^{\gamma x} z(x)$ is directly Riemann integrable and Fatou's lemma, we have

$$
q I\left(1-q g_{W}(-\gamma)\right)^{-2} \bar{W}(x) \lesssim Z_{3}(x) .
$$


Combining this with (5.10), (5.13), and (5.14), we obtain

$$
Z_{2}(x)=o(1) \bar{W}(x) .
$$

By $U_{0}(\mathrm{~d} y)=(1-q) \sum_{n=1}^{\infty} q^{n} W^{* n}(\mathrm{~d} y)$ and (5.19), we obtain

$$
\int_{h(x)}^{x-h(x)} \bar{W}(x-y) W(\mathrm{~d} y)=O(1) \int_{h(x)}^{x-h(x)} \bar{W}(x-y) U_{0}(\mathrm{~d} y)=o(1) \bar{W}(x) .
$$

Hence, together with $W \in \mathcal{L}(\gamma)$, this implies that $W \in \delta(\gamma)$.

Case 2. Its proof is similar to the proof of case 2 of Theorem 5.1. We omit the details.

Remark 5.1. In case 2 of Theorem 5.2, if $\bar{W}(x)=o(1) \bar{L}(x)$ and $L \in \delta(\gamma)$, it is easy to show that $\overline{U_{0}}(x)=o(1) \overline{L(x)}$. Hence, (5.11) still holds.

\section{Proofs of the main results}

In this section we will use the results of Sections 4 and 5 to prove the theorems of Section 2. Using renewal equations and the strong Markov property, we first investigate the relationships between the moments of the overshoot and undershoot and the moments of the first ascending ladder height of a random walk.

Let $T$ be any positive constant. If we write

$$
Z(x)=\mathrm{E} \varphi\left(S_{\tau_{x}}-x\right) \mathbf{1}_{\left\{S_{\tau_{x}} \in x+\Delta_{T}\right\}}, \quad z(x)=\mathrm{E} \varphi\left(S_{\tau_{+}}-x\right) \mathbf{1}_{\left\{S_{\tau_{+}} \in x+\Delta_{T}\right\}}, \quad \text { and } \quad W=F_{p+},
$$

then, by the strong Markov property,

$$
\begin{aligned}
Z(x) & =\mathrm{E} \varphi\left(S_{\tau_{x}}-x\right) \mathbf{1}_{\left\{\left(S_{\tau_{x}} \in x+\Delta_{T}\right), S_{\tau_{+}}>x\right\}}+\mathrm{E} \varphi\left(S_{\tau_{x}}-x\right) \mathbf{1}_{\left\{\left(S_{\tau_{x}} \in x+\Delta_{T}\right), S_{\tau_{+}} \leq x\right\}} \\
& =\mathrm{E} \varphi\left(S_{\tau_{+}}-x\right) \mathbf{1}_{\left\{S_{\tau_{+}} \in x+\Delta_{T}\right\}}+\int_{0}^{x} \mathrm{E} \varphi\left(S_{\tau_{x-y}}-(x-y)\right) \mathbf{1}_{\left\{S_{\tau_{x-y} \in x-y+\Delta_{T}}\right\}} F_{+}(\mathrm{d} y) \\
& =z(x)+\alpha \int_{0}^{x} Z(x-y) W(\mathrm{~d} y)
\end{aligned}
$$

Similarly, if we write

$$
\begin{gathered}
Z(x)=\mathrm{E} \varphi\left(x-S_{\tau_{x}-1}\right) \mathbf{1}_{\left\{S_{\tau_{x}} \in x+\triangle_{T}\right\}}, \quad z(x)=\mathrm{E} \varphi\left(x-S_{\tau_{+}-1}\right) \mathbf{1}_{\left\{S_{\tau_{+}} \in x+\triangle_{T}\right\}}, \\
\text { and } W=F_{p+} ; \\
Z(x)=\mathrm{E} \varphi\left(S_{\tau_{x}}-x\right) \mathbf{1}_{\left\{\tau_{x}<\infty\right\}}, \quad z(x)=\mathrm{E} \varphi\left(S_{\tau_{+}}-x\right) \mathbf{1}_{\left\{S_{\tau_{+}}>x\right\}}, \quad \text { and } \quad W=F_{p+} ;
\end{gathered}
$$

and

$$
Z(x)=\mathrm{E} \varphi\left(x-S_{\tau_{x}-1}\right) \mathbf{1}_{\left\{\tau_{x}<\infty\right\}}, \quad z(x)=\mathrm{E} \varphi\left(x-S_{\tau_{+}-1}\right) \mathbf{1}_{\left\{S_{\tau_{+}}>x\right\}}, \quad \text { and } \quad W=F_{p+},
$$

then we obtain three renewal equations similar to (6.1), denoting them by (6.2), (6.3), and (6.4), respectively.

Proof of Theorem 2.1. We use the renewal equation (6.1). Since $F \in \mathcal{L}$, then $F^{I} \in \mathcal{L}_{\triangle_{T}}$ for all $0<T \leq \infty$. Hence, by Theorem 4.2(ii), the dominated convergence theorem, and 
Lemma 3 of Asmussen et al. (2002), we have, for any $0<T<\infty$,

$$
\begin{aligned}
z(x) & =\mathrm{E} \varphi\left(S_{\tau_{+}}-x\right) \mathbf{1}_{\left\{S_{\tau_{+}} \in x+\triangle_{T}\right\}} \\
& \sim(1-\alpha) m^{-1} \int_{0}^{T} \varphi(y) \bar{F}(y+x) \mathrm{d} y \\
& \sim(1-\alpha) m^{-1} \int_{0}^{T} \varphi(y) \mathrm{d} y \bar{F}(x) \\
& \sim(1-\alpha) m^{-1} \int_{0}^{T} \varphi(y) \mathrm{d} y T^{-1} F^{I}\left(x+\triangle_{T}\right) \\
& \sim T^{-1} \int_{0}^{T} \varphi(y) \mathrm{d} y F_{+}\left(x+\triangle_{T}\right) .
\end{aligned}
$$

Hence, by (6.1), Theorem 5(ii) of Asmussen et al. (2003), and Theorem 2.2 of Wang et al. (2008), it follows that (ii) is equivalent to (iii). The assertion that (i) is equivalent to (ii) follows from $F \in \mathcal{L}$ and Lemma 4.2 of Wang et al. (2007).

Proof of Theorem 2.2. We use the renewal equation (6.2). By Theorem 4.3(ii) we know that (4.6) holds.

Case 1. By (4.6), $0<a<\infty, F^{I} \in \varsigma_{\triangle_{T}}$ for all $0<T<\infty$, and Lemma 3.3 of Asmussen et al. (2002), we obtain

$$
\begin{aligned}
z(x)=\operatorname{E} \varphi\left(x-S_{\tau_{+}-1}\right) \mathbf{1}_{\left\{S_{\tau_{+}} \in x+\triangle_{T_{1}}\right\}} & \sim(1-\alpha) m^{-1} a F^{I}\left(x+\triangle_{T_{1}}\right) \\
& \sim a F_{+}\left(x+\triangle_{T_{1}}\right) .
\end{aligned}
$$

Hence, by Theorem 5(ii) of Asmussen et al. (2003) and (5.4), (2.3) can be proved.

Case 2. It follows from (4.6) and $\varphi(x) \rightarrow \infty$ that

$$
F^{I}\left(x+\triangle_{T_{1}}\right)=o(1) z(x)
$$

Hence, by Theorem 5(iii) of Asmussen et al. (2003),

$$
\begin{aligned}
Z(x)=\operatorname{E} \varphi\left(x-S_{\tau_{x}-1}\right) \mathbf{1}_{\left\{S_{\tau_{x}} \in x+\Delta_{T_{1}}\right\}} & \sim(1-\alpha)^{-1} z(x) \\
& \sim m^{-1} \int_{x}^{\infty} \varphi(y) F\left(y+\triangle_{T_{1}}\right) \mathrm{d} y,
\end{aligned}
$$

i.e. (2.4) holds.

Proof of Theorem 2.3. We use the renewal equation (6.3).

Case 1. It can be immediately proved by Theorem 4.2(i), Corollary 5.1, and (6.3).

Case 2. For any $N>0$, since $a=\infty$, there exists a $b>0$ such that

$$
\bar{G}(x) \geq \int_{b}^{\infty} \varphi(y) \bar{F}(x+y) \mathrm{d} y \geq N \overline{F^{I}}(x+b) .
$$

Since $F^{I} \in \mathcal{L}$, then we have $\overline{F^{I}}(x)=o(1) \bar{G}(x)$. Hence, $\overline{U_{0}}(x)=o(1) \bar{G}(x)$. So (2.7) can be obtained from (6.3), Theorem 4.2(i), and case 2 of Theorem 5.1. 
Proof of Theorem 2.4. We use the renewal equation (6.4).

Case 1. By Theorem 4.3(i), (4.5) still holds. By (4.5) and $\varphi(x) \rightarrow a, 0<a<\infty$, we obtain

$$
\begin{aligned}
z(x)=\operatorname{E} \varphi\left(x-S_{\tau_{+}-1}\right) \mathbf{1}_{\left\{S_{\tau_{+}}>x\right\}} & \sim(1-\alpha) m^{-1} a \overline{F^{I}}(x) \\
& \sim a \alpha \bar{F}_{p+}(x) \\
& =\alpha a \bar{W}(x) .
\end{aligned}
$$

Hence, it follows from Corollary 5.1 and (6.4) that (i) is equivalent to (ii).

Case 2. By Theorem 4.3(i) and $F^{I} \in \mathcal{L},(4.5)$ still holds.

It follows from $a=\infty$ that $\overline{F^{I}}(x)=o(1) \bar{G}(x)$. Since $G \in \&$, by Theorem 2(i) of Asmussen et al. (2003), $\overline{U_{0}}(x)=o(1) \bar{G}(x)$. Hence, (2.9) can be shown similarly to case 2 of Theorem 2.3 using (6.4).

Proof of Theorem 2.5. We use the renewal equation (6.3). Since $\varphi \in \mathcal{L} d$, then it is easy to show that $\int_{0}^{\infty} \varphi(y) \mathrm{e}^{-\gamma y} \mathrm{~d} y<\infty$. It follows from $\bar{F}(x) \mathrm{e}^{\gamma x} \downarrow$ that, for $x>0$,

$$
\int_{0}^{\infty} \varphi(y) \bar{F}(x+y) \mathrm{d} y \leq C \int_{0}^{\infty} \varphi(y) \mathrm{e}^{-\gamma y} \mathrm{~d} y \bar{F}(x) .
$$

Hence, by Theorem 4.5, $f \in \mathcal{L} d(\gamma)$, and the dominated convergence theorem,

$$
\begin{aligned}
z(x) & =\mathrm{E} \varphi\left(S_{\tau_{+}}-x\right) \mathbf{1}_{\left\{S_{\tau_{+}}>x\right\}} \\
& \sim\left(1-g_{-}(-\gamma)\right)^{-1} \int_{x}^{\infty} \varphi(y-x) f(y) \mathrm{d} y \\
& \sim \gamma\left(1-g_{-}(-\gamma)\right)^{-1} \int_{0}^{\infty} \varphi(y) \bar{F}(x+y) \mathrm{d} y \\
& \sim \gamma \int_{0}^{\infty} \varphi(y) \mathrm{e}^{-\gamma y} \mathrm{~d} y \bar{F}_{+}(x) .
\end{aligned}
$$

Hence, the result can be proved using case 1 of Theorem 5.2.

Proof of Theorem 2.6. We use the renewal equation (6.4).

Case 1. By Theorem 4.4,

$$
\begin{aligned}
z(x)=\operatorname{E} \varphi\left(x-S_{\tau_{+}-1}\right) \mathbf{1}_{\left\{S_{\tau_{+}}>x\right\}} & \sim\left(1-g_{-}(-\gamma)\right)^{-1} a \bar{F}(x) \\
& \sim \alpha a \bar{F}_{p+}(x)
\end{aligned}
$$

Hence, by case 1 of Theorem 5.2, the result can be obtained.

Case 2. It follows from $a=\infty$ that $\bar{F}_{p+}=o(1) \bar{G}(x)$. Also, by $G \in \delta(\gamma), \bar{U}_{0}(x)=$ $o(1) \bar{G}(x)$. Hence, (2.10) can be shown using case 2 of Theorem 5.2.

\section{Acknowledgements}

The authors wish to thank the anonymous referees and the Editor for their very valuable comments on an earlier version of this paper. This research was supported by the National Science Foundation of China (grant number 10671139). 


\section{References}

Asmussen, S. (1998). A probabilistic look at the Wiener-Hopf equation. SIAM Rev. 40, 189-201.

Asmussen, S. (2003). Applied Probability and Queues (Appl. Math. 51), 2nd edn. Springer, New York.

Asmussen, S., Foss, S. AND Korshunov, D. (2003). Asymptotics for sums of random variables with local subexponential behavior. J. Theoret. Prob. 16, 489-518.

Asmussen, S. et al. (2002). A local limit theorem for random walk maxima with heavy tails. Statist. Prob. Lett. 56, 399-404.

Bertoin, J. And Doney, R. A. (1996). Some asymptotic results for transient random walks. Adv. Appl. Prob. 28, 207-226.

Borovkov, A. A. (1976). Stochastic Processes in Queueing Theory (Appl. Math. 4). Springer, Berlin.

BorovKov, A. A. AND Foss, S. (2000). Estimates for overshooting an arbitrary boundary by a random walk and their applications. Theory Prob. Appl. 44, 231-253.

CAI, J. AND Garrido, J. (2002). Asymptotic forms and bounds for tails of convolutions of compound geometric distributions, with applications. In Recent Advances in Statistical Methods, ed. Y. P. Chaubey, Imperial College Press, London, pp. 114-131.

CAI, J. AND TANG, Q. (2004). On max-sum equivalence and convolution closure of heavy-tailed distributions and their applications. J. Appl. Prob. 41, 117-130.

Cheng, Y., Tang, Q. and Yang, H. (2002). Approximations for moments of deficit at ruin with exponential and subexponential claims. Statist. Prob. Lett. 59, 367-378.

Chen, G., Wang, Y. And Cheng, F. (2009). The uniform local asymptotics of the overshoot of a random walk with heavy-tailed increments. To appear in Stoch. Models.

Chistyakov, V. P. (1964). A theorem on sums of independent positive random variables and its applications to branching process. Theory Prob. Appl. 9, 640-648.

Chover, J., Ney, P. AND Wainger, S. (1973a). Degeneracy properties of subcritical branching processes. Ann. Prob. 1, 663-673.

Chover, J., Ney, P. And Wainger, S. (1973b). Functions of probability measures. J. Analyse Math. 26, 255-302.

Cuine, D. B. H. (1987). Convolutions of distributions with exponential and subexponential tails. J. Austral. Math. Soc. Ser. A 43, 347-365.

CUI, Z. AND WANG, Y. (2007). A simple proof of approximations for moments of deficit at ruin. J. Suzhou Univ. 23, 23-25 (in Chinese).

Denisov, D. AND ShNEER, V. (2007). Local asymptotics of the cycle maximum of a heavy-tailed random walk. Adv. Appl. Prob. 39, 221-244.

Denisov, D., Foss, S. AND Korshunov, D. (2004). Tail asymptotics for the supremum of a random walk when the mean is not finite. Queueing Systems 46, 15-33.

Doney, R. A. And Kyprianou, A. E. (2006). Overshoots and undershoots of Lévy processes. Ann. Appl. Prob. 16, 91-106.

Embrechts, P. AND Goldie, C. M. (1982). On convolution tails. Stoch. Process. Appl. 13, 263-278.

Embrechts, P., Goldie, C. M. and VeraverbeKe, N. (1979). Subexponentiality and infinite divisibility. Z. Wahrscheinlichkeitsth. 49, 335-347.

Embrechts, P., KlÜPpelberg, C. AND Miкosch, T. (1997). Modelling Extremal Events (Appl. Math. 33). Springer, Berlin.

Foss, S. And Korshunov, D. (2007). Lower limits and equivalences for convolution tails. Ann. Prob. 35, 366-383.

Foss, S. AND Zachary, S. (2003). The maximum on a random time interval of a random walk with long-tailed increments and negative drift. Ann. Appl. Prob. 13, 37-53.

GAO, Q. AND WANG, Y. (2009). Ruin probability and local ruin probability in the random multi-delayed renewal risk model. Statist. Prob. Lett. 79, 588-596.

JANSON, S. (1986). Moments for first-passage and last-exit times, the minimum, and related quantities for random walks with positive drift. Adv. Appl. Prob. 18, 865-879.

KlÜPPELberG, C. (1988). Subexponential distributions and integrated tails. J. Appl. Prob. 25, 132-141.

KLÜPPELBerg, C. (1989). Subexponential distributions and characterizations of related classes. Prob. Theory Relat. Fields 82, 259-269.

KlüPpelberg, C., KyPRianou, A. E. and Maller, R. A. (2004). Ruin probabilities and overshoots for general Lévy insurance risk processes. Ann. Appl. Prob. 14, 1766-1801.

Pakes, A. G. (2004). Convolution equivalence and infinite divisibility. J. Appl. Prob. 41, 407-424.

PARK, H. S. AND MALLER, R. (2008). Moment and MGF convergence of overshoots and undershoots for Lévy insurance risk processes. Adv. Appl. Prob. 40, 716-733.

TANG, Q. (2007). The overshoot of a random walk with negative drift. Statist. Prob. Lett. 77, 158-165.

Veraverbeke, N. (1977). Asymptotic behaviour of Wiener-Hopf factors of a random walk. Stoch. Process. Appl. 5, $27-37$. 
WANG, K., WANG, Y. AND YIN, C. (2008). Equivalent conditions of local asymptotics for the overshoot of a random walk with heavy-tailed increments. Submitted.

WANG, Y. AND WANG, K. (2006). Asymptotics of the density of the supremum of a random walk with heavy-tailed increments. J. Appl. Prob. 43, 874-879.

WANG, Y. AND WANG, K. (2009). Equivalent conditions of asymptotics for the density of the supremum of a random walk in the intermediate case. J. Theoret. Prob. 22, 281-293.

WANG, Y., CHENG, D. AND WANG, K. (2005). The closure of a local subexponential distribution class under convolution roots with applications to the compound Poisson process. J. Appl. Prob. 42, 1194-1203.

WANG, Y., YANG, Y., WANG, K. And Cheng, D. (2007). Some new equivalent conditions on asymptotics and local asymptotics for random sums and their applications. Insurance Math. Econom. 40, 256-266.

Yin, C. AND ZHaO, J. (2006). Nonexponential asymptotics for the solutions of renewal equations, with applications. J. Appl. Prob. 43, 815-824.

Yu, C., Wang, Y. AND CuI, Z. (2009). Lower limits and upper limits for tails of random sums supported on R. Submitted. 